\title{
Fisheries management in random environments: comparison of harvesting policies for the logistic model \\ Supplementary material
}

Nuno M. Brites ${ }^{1}$ (corresponding author brites@uevora.pt)

Carlos A. Braumann ${ }^{1,2}$ (braumann@uevora.pt)

July 17, 2017

\footnotetext{
${ }^{1}$ Centro de Investigação em Matemática e Aplicações, Instituto de Investigação

e Formação Avançada, Universidade de Évora, Évora, 7000-671, Portugal

${ }^{2}$ Departamento de Matemática, Escola de Ciências e Tecnologia, Universidade de Évora, Évora, 7000-671, Portugal
} 


\section{Supplementary figures showing the time evo- lution of population size, effort and profit per unit time for scenarios $S_{1}$ to $S_{14}$}

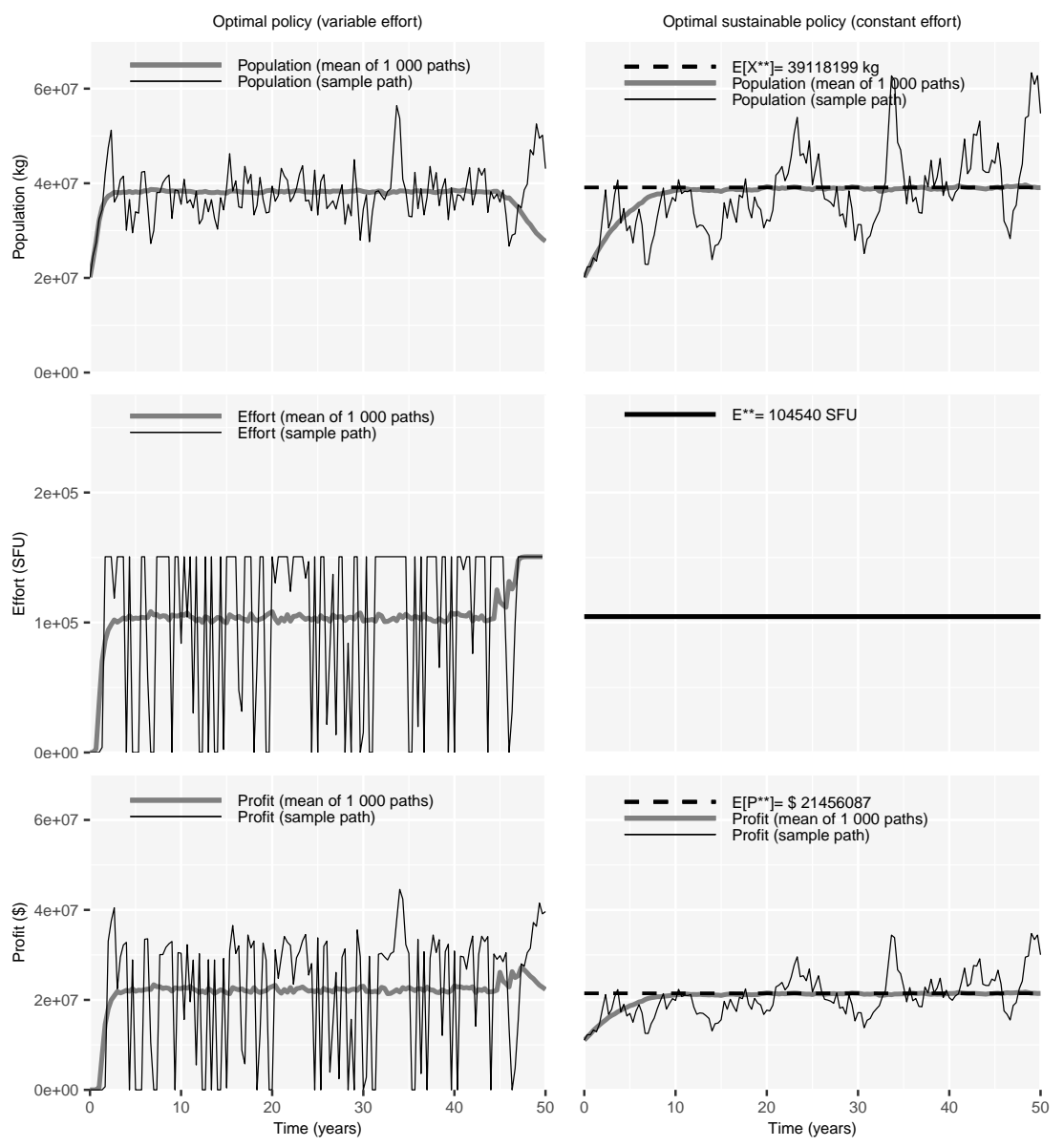

Figure 1: Scenario $S_{1}$ : mean and randomly chosen sample path for the population, the effort and the profit per unit time. The optimal variable effort policy is on the left side and the optimal constant effort sustainable policy is on the right side. 


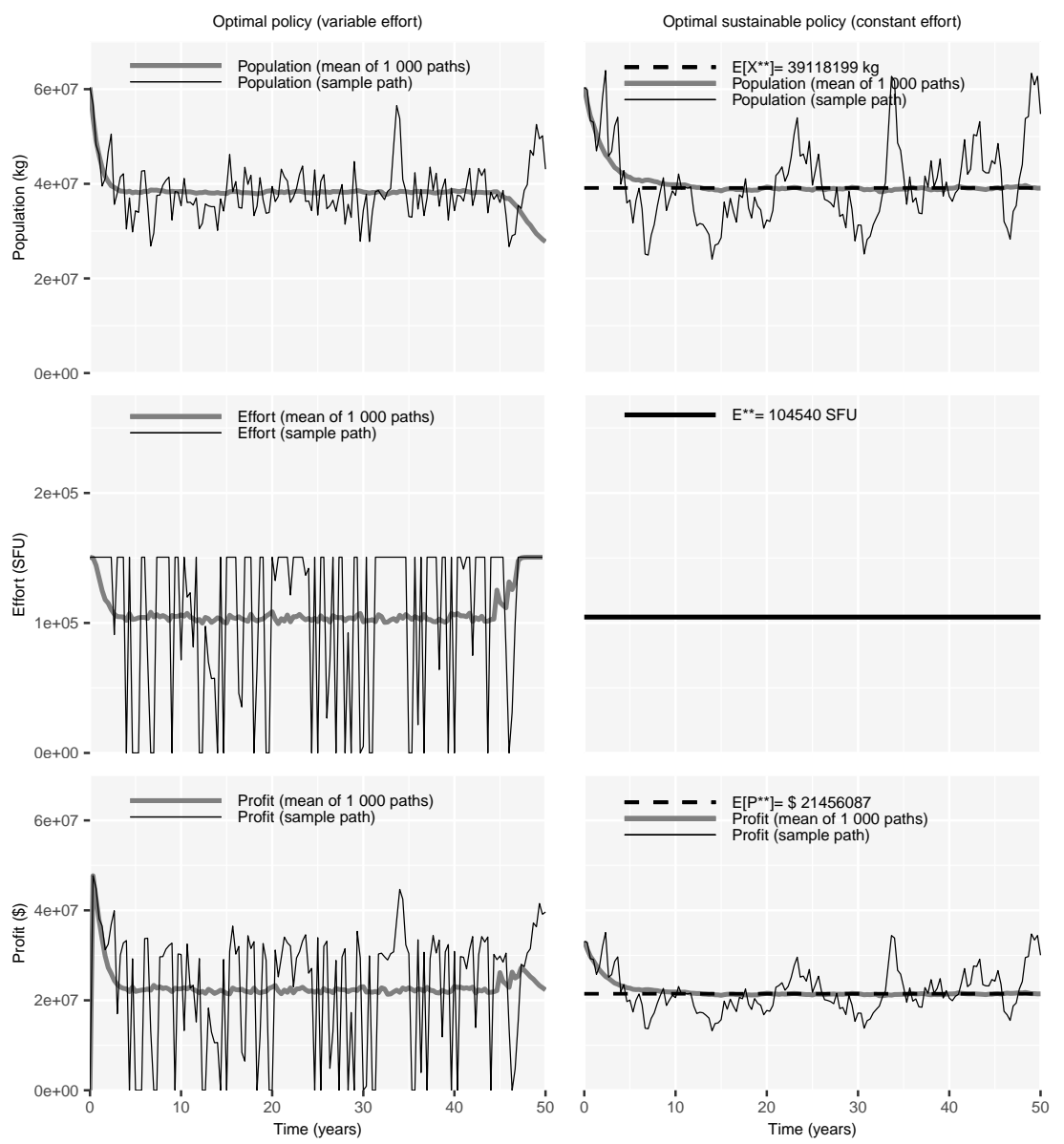

Figure 2: Scenario $S_{2}$ : mean and randomly chosen sample path for the population, the effort and the profit per unit time. The optimal variable effort policy is on the left side and the optimal constant effort sustainable policy is on the right side. 

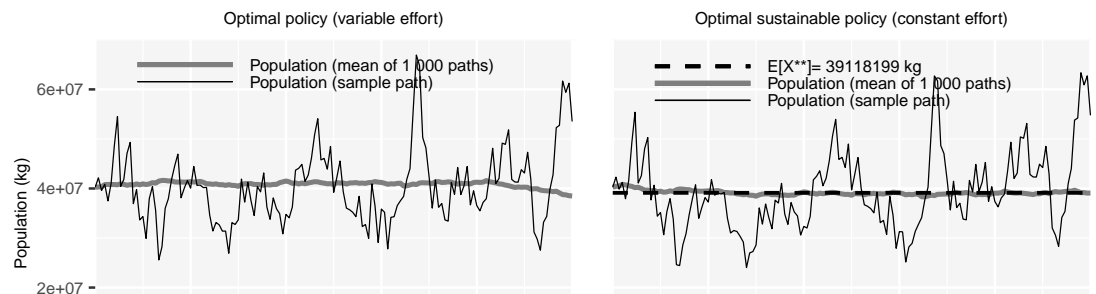

$0 \mathrm{e}+00-$
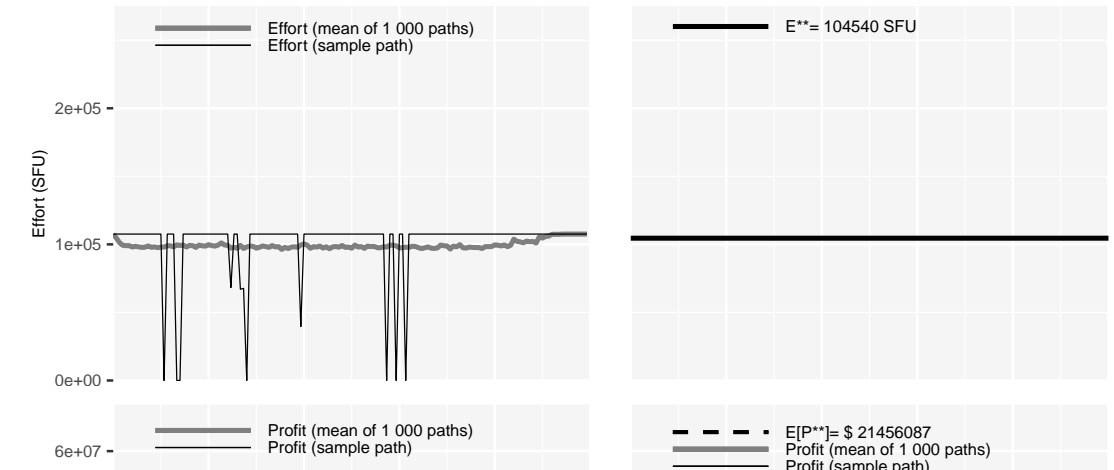

- - - E E $\left[P^{* *}\right]=\$ 21456087$ Profit (mean of 1000 paths)

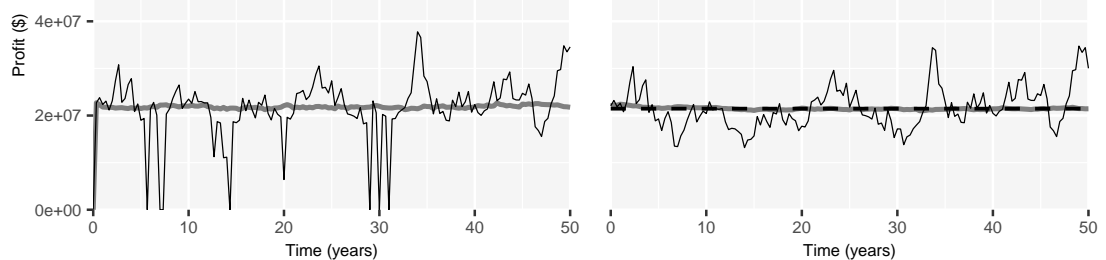

Figure 3: Scenario $S_{3}$ : mean and randomly chosen sample path for the population, the effort and the profit per unit time. The optimal variable effort policy is on the left side and the optimal constant effort sustainable policy is on the right side. 

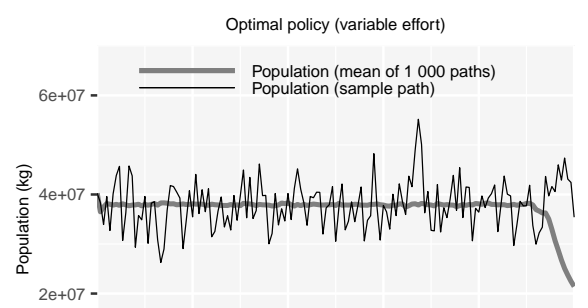

$0 \mathrm{e}+00-$
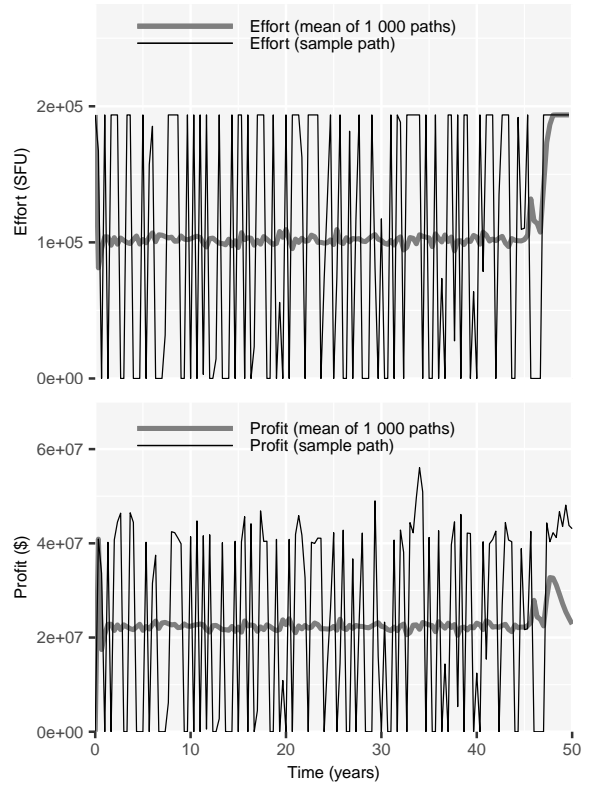
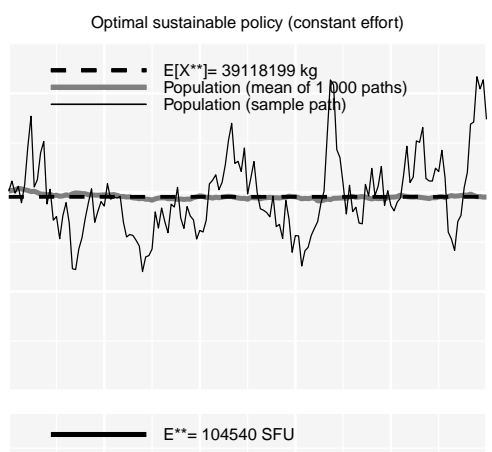

- - - $E\left[P^{* *}\right]=\$ 21456087$ Profit (mean of 1000 paths)
Profit (sample path)

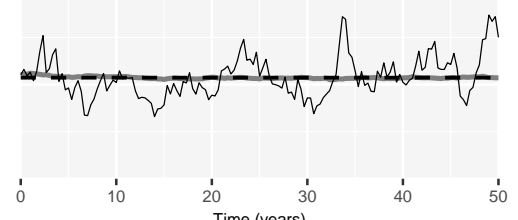

Figure 4: Scenario $S_{4}$ : mean and randomly chosen sample path for the population, the effort and the profit per unit time. The optimal variable effort policy is on the left side and the optimal constant effort sustainable policy is on the right side. 

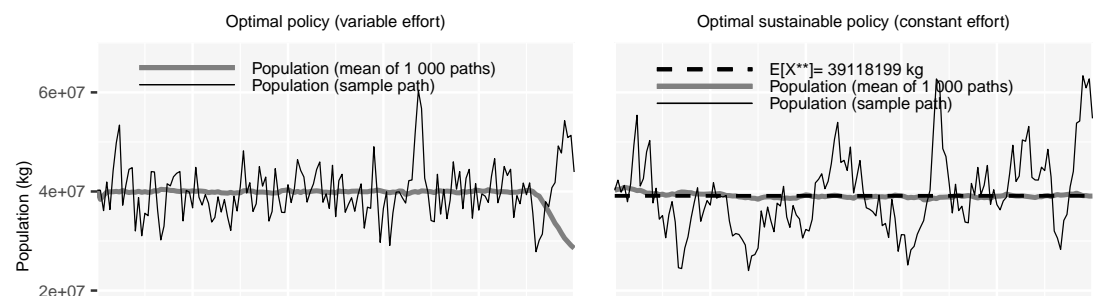

$0 \mathrm{e}+00$ -
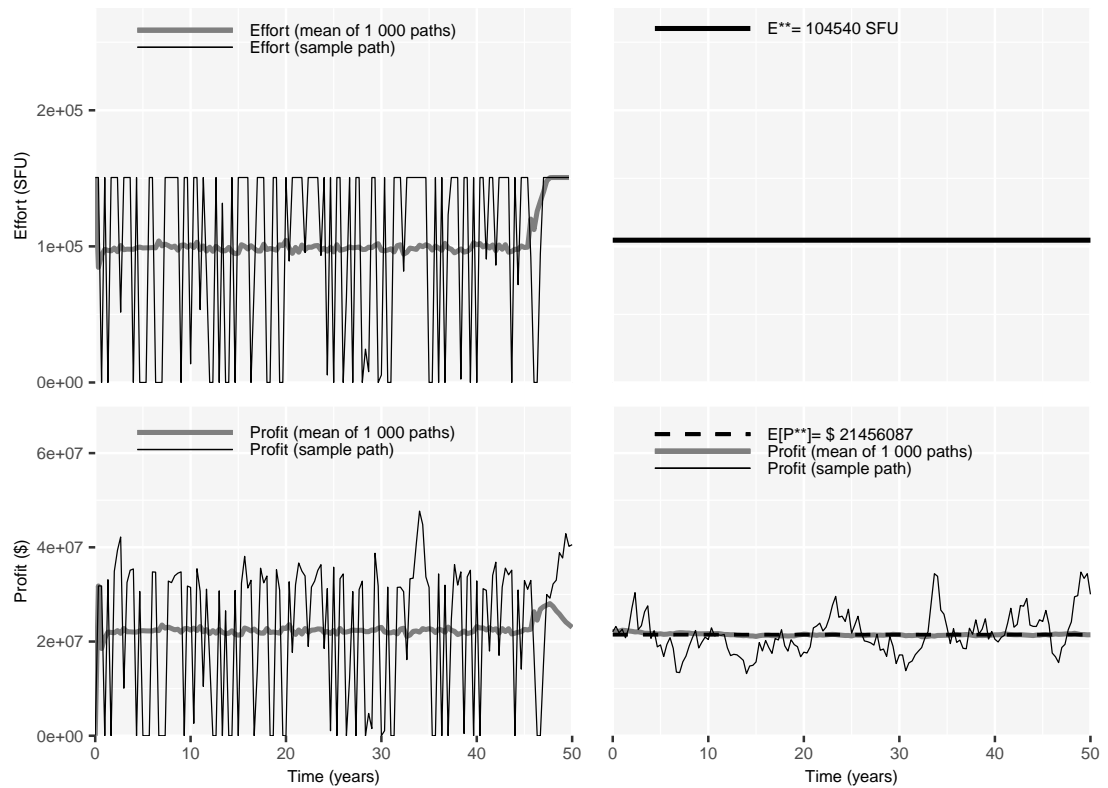

Figure 5: Scenario $S_{5}$ : mean and randomly chosen sample path for the population, the effort and the profit per unit time. The optimal variable effort policy is on the left side and the optimal constant effort sustainable policy is on the right side. 


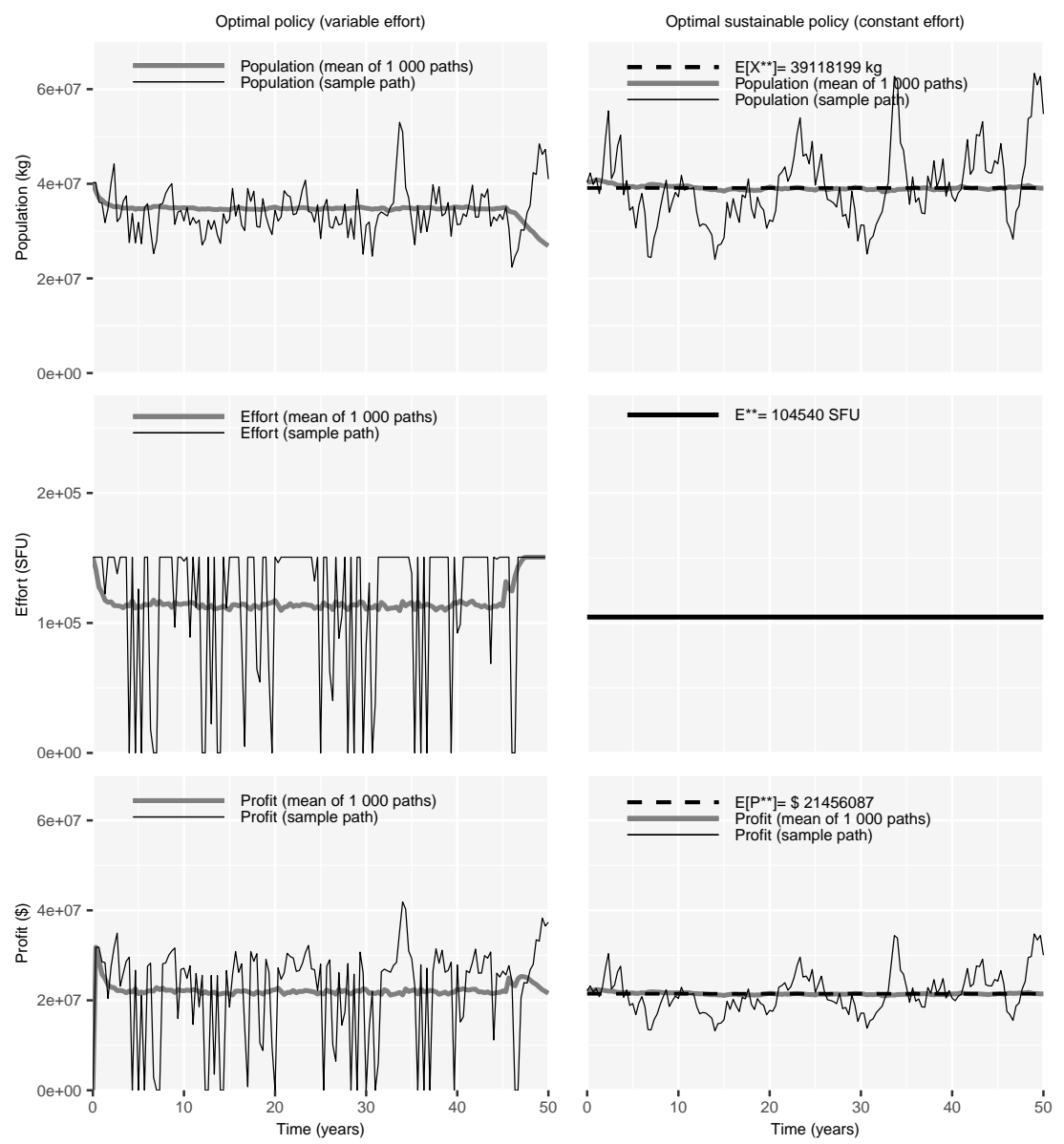

Figure 6: Scenario $S_{6}$ : mean and randomly chosen sample path for the population, the effort and the profit per unit time. The optimal variable effort policy is on the left side and the optimal constant effort sustainable policy is on the right side. 

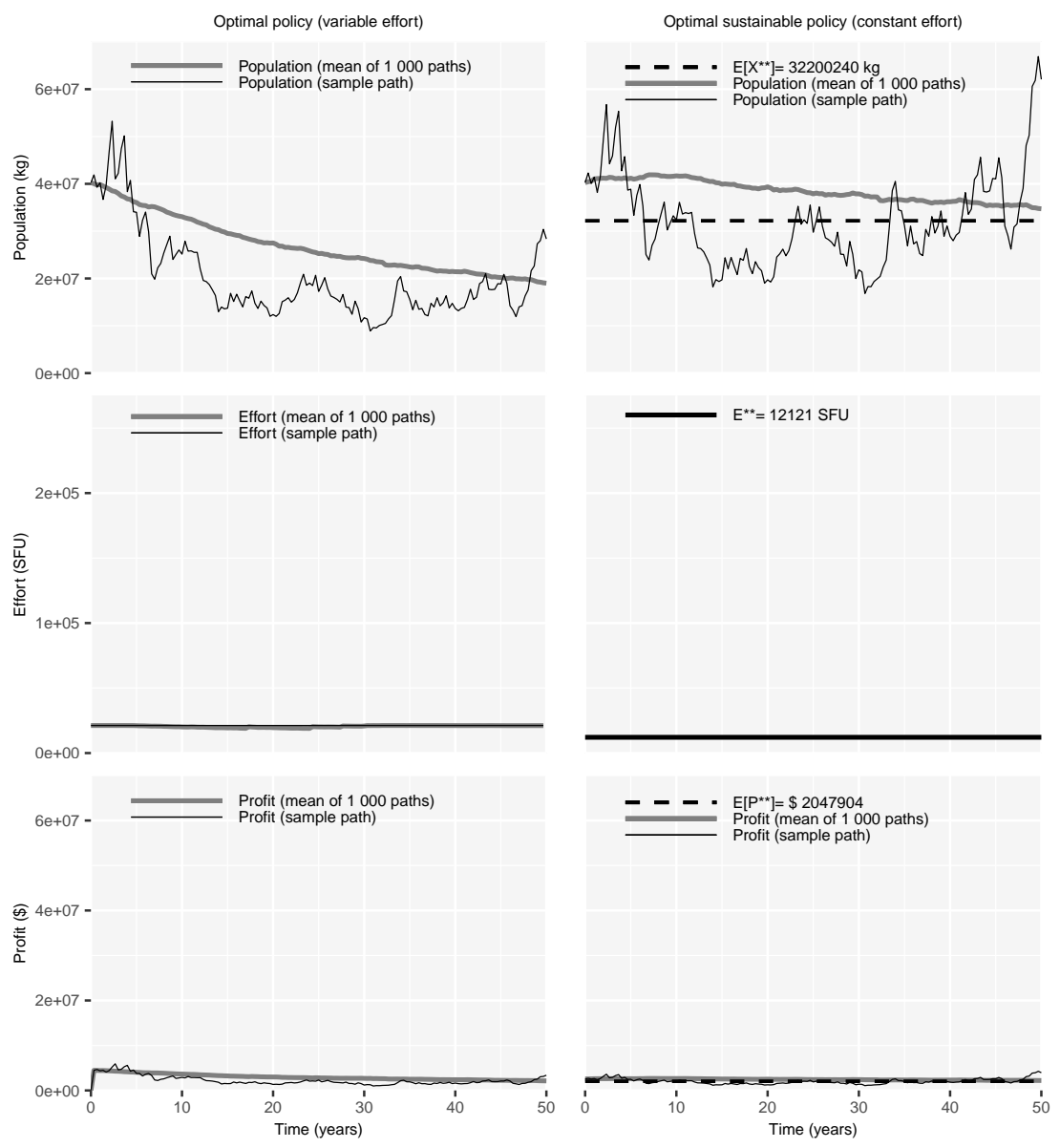

Figure 7: Scenario $S_{7}$ : mean and randomly chosen sample path for the population, the effort and the profit per unit time. The optimal variable effort policy is on the left side and the optimal constant effort sustainable policy is on the right side. 


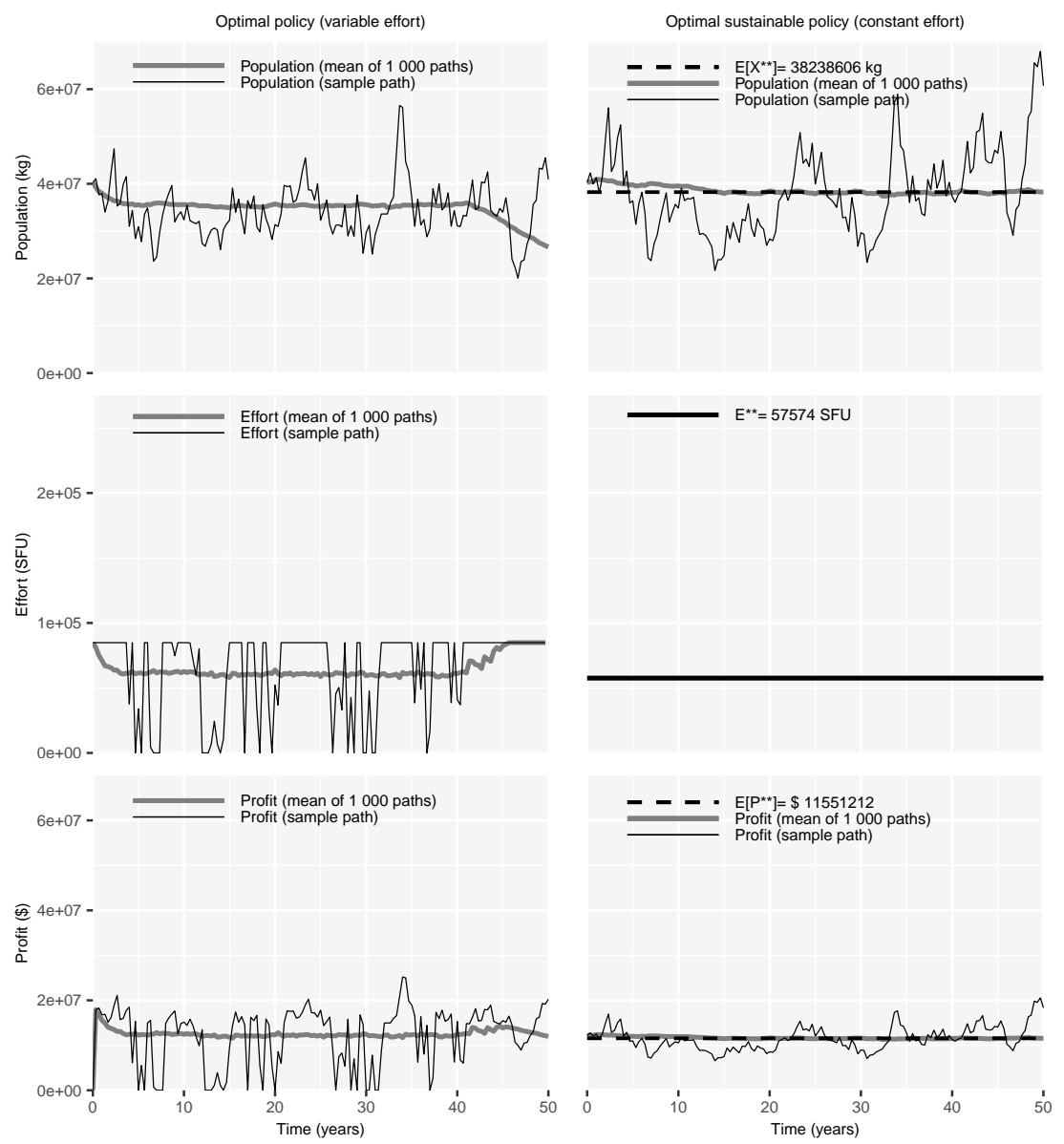

Figure 8: Scenario $S_{8}$ : mean and randomly chosen sample path for the population, the effort and the profit per unit time. The optimal variable effort policy is on the left side and the optimal constant effort sustainable policy is on the right side. 


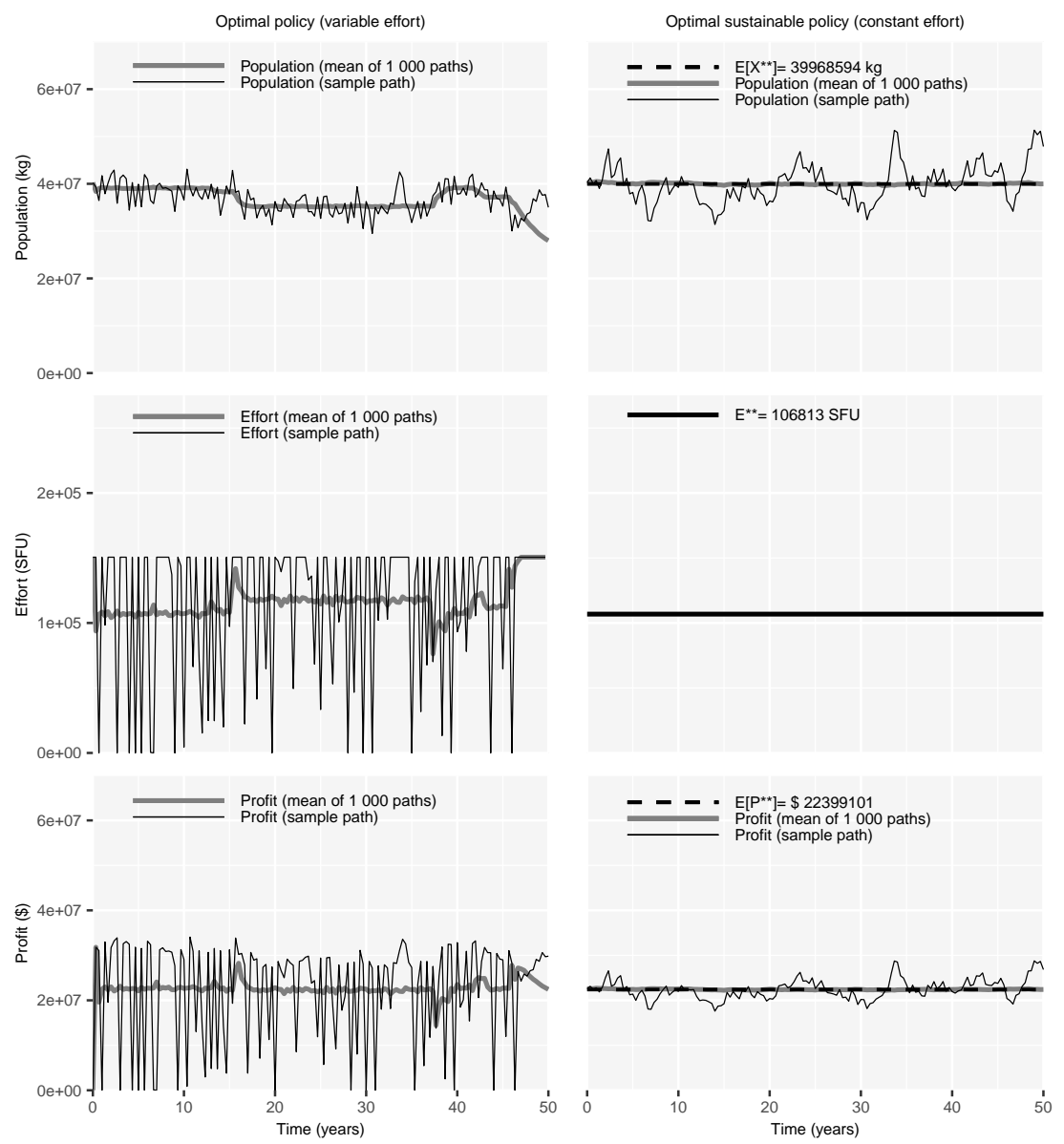

Figure 9: Scenario $S_{9}$ : mean and randomly chosen sample path for the population, the effort and the profit per unit time. The optimal variable effort policy is on the left side and the optimal constant effort sustainable policy is on the right side. 

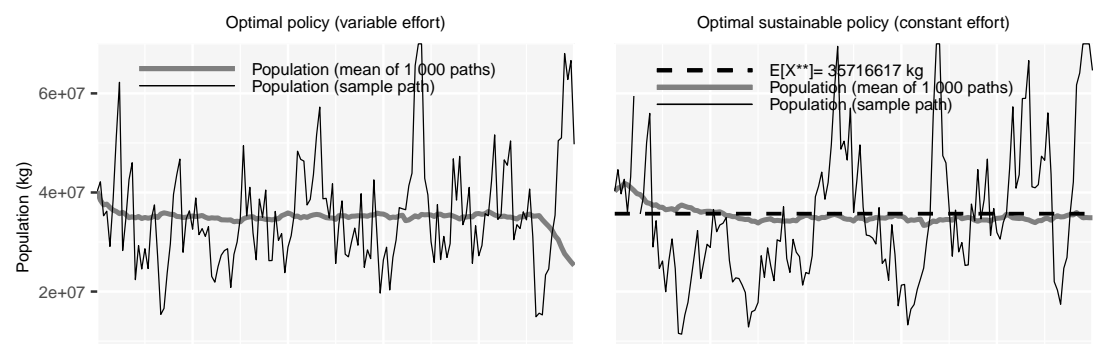

$0 \mathrm{e}+00-$
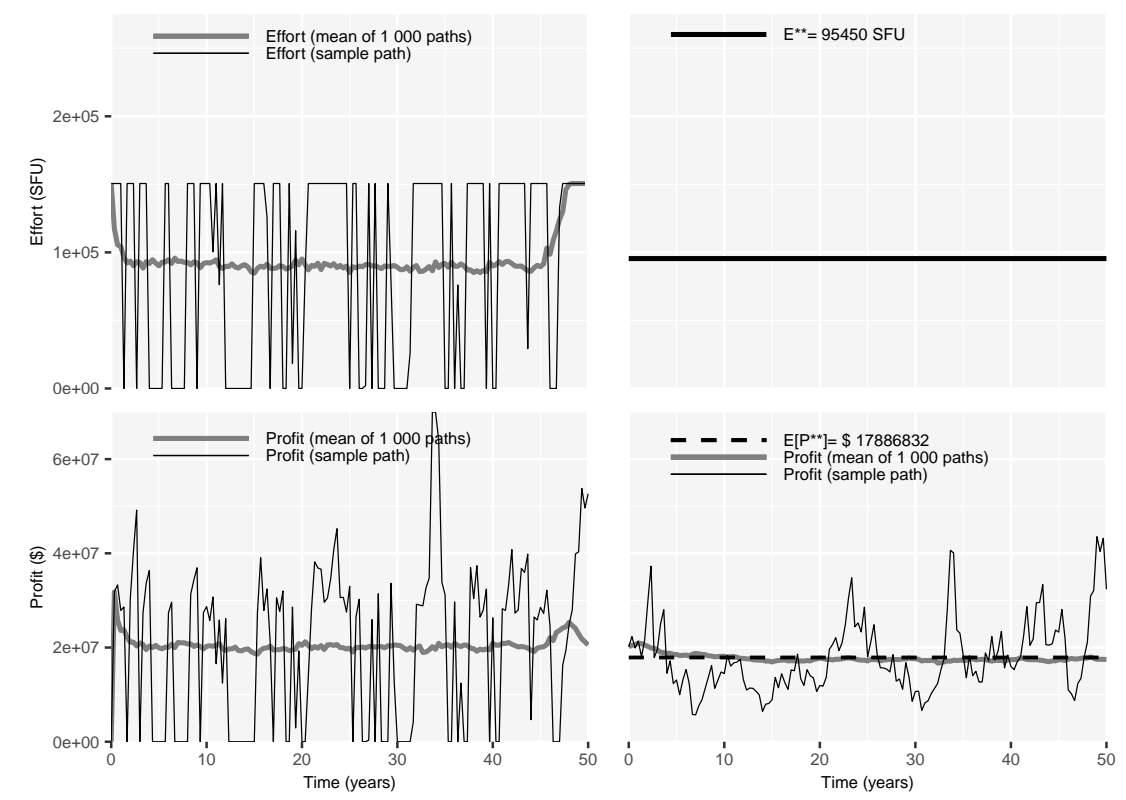

Figure 10: Scenario $S_{10}$ : mean and randomly chosen sample path for the population, the effort and the profit per unit time. The optimal variable effort policy is on the left side and the optimal constant effort sustainable policy is on the right side. 


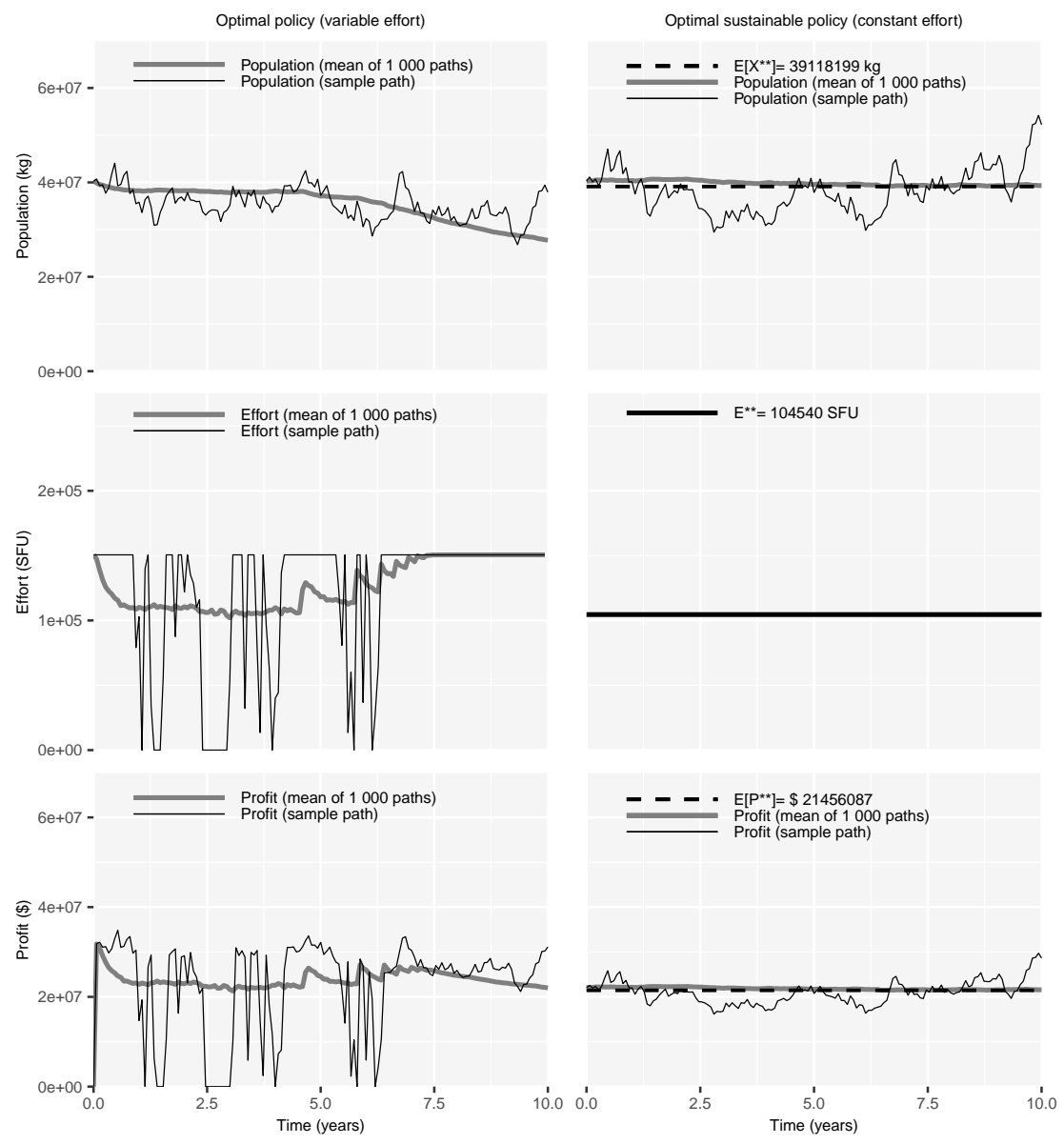

Figure 11: Scenario $S_{11}$ : mean and randomly chosen sample path for the population, the effort and the profit per unit time. The optimal variable effort policy is on the left side and the optimal constant effort sustainable policy is on the right side. 


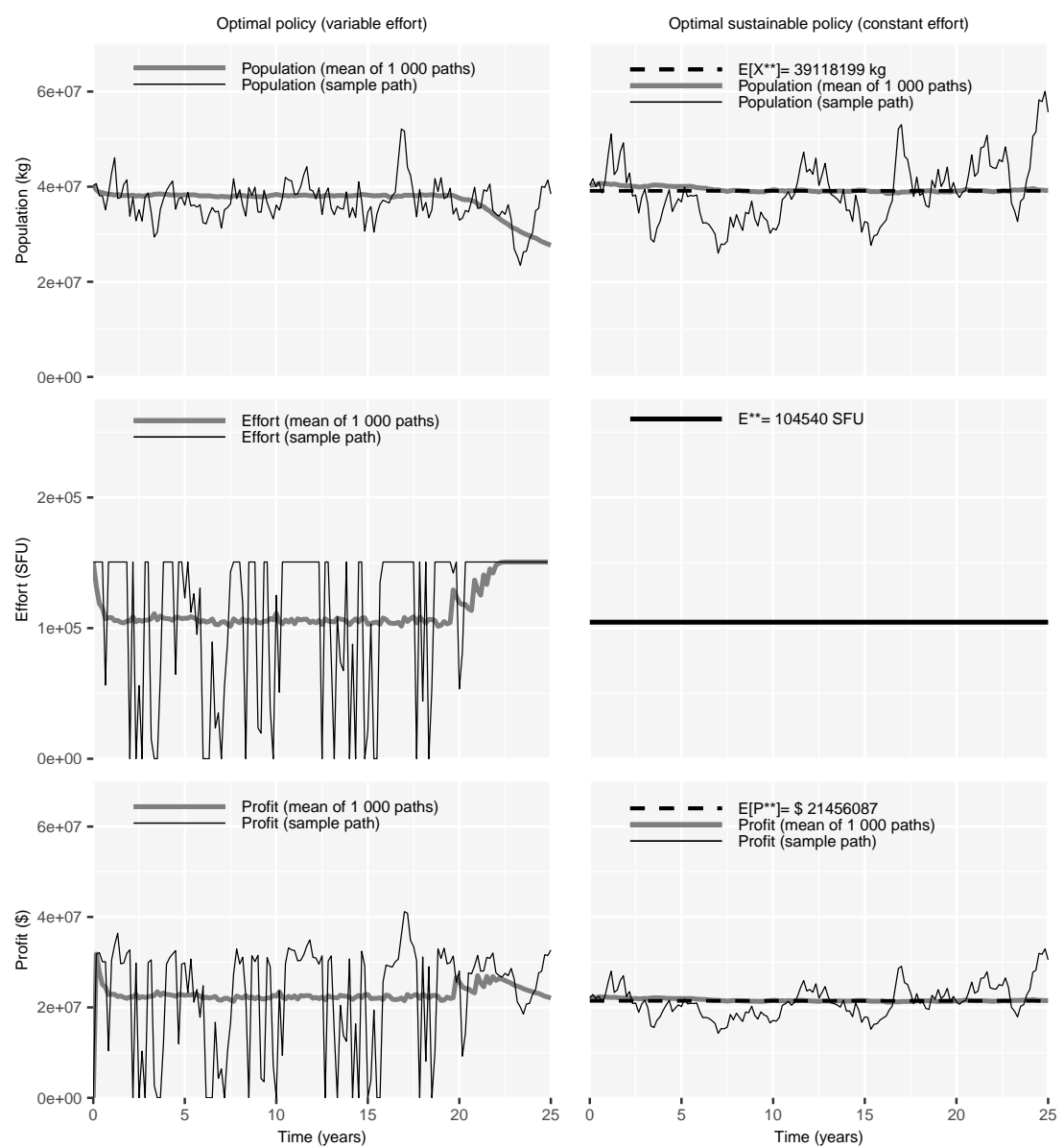

Figure 12: Scenario $S_{12}$ : mean and randomly chosen sample path for the population, the effort and the profit per unit time. The optimal variable effort policy is on the left side and the optimal constant effort sustainable policy is on the right side. 

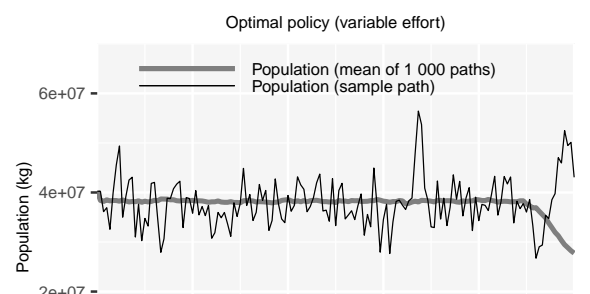

$0 \mathrm{e}+00-$

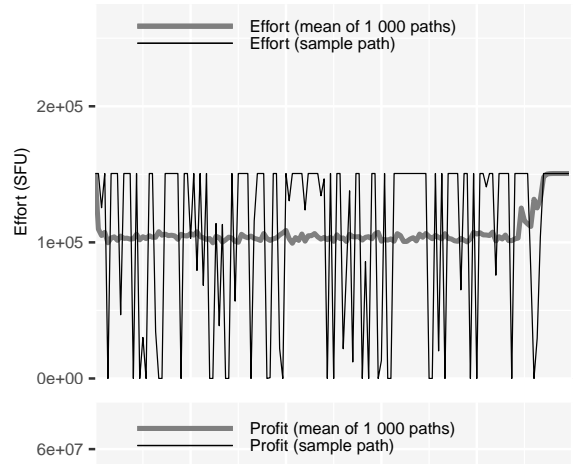

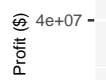

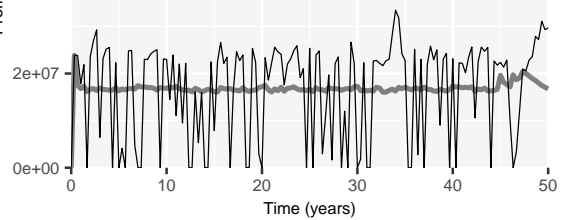

Optimal sustainable policy (constant effort)

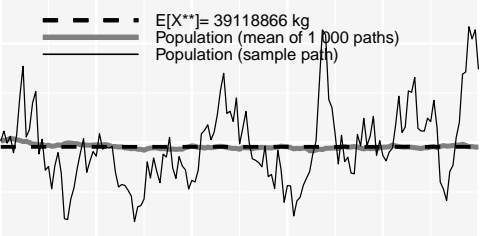

$\left[E^{\star *}=104538 \mathrm{SFU}\right.$

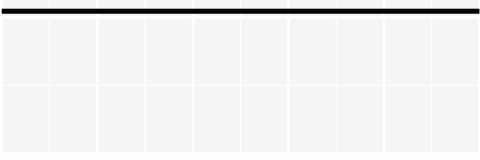

- - - $E\left[P^{* *}\right]=\$ 16091790$ Profit (mean of 1000 paths)

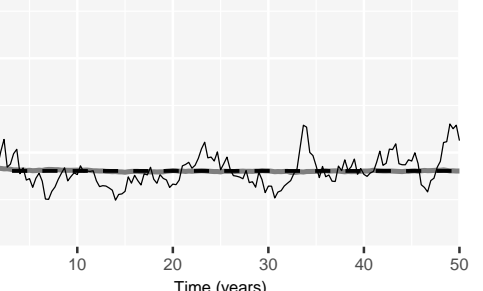

Figure 13: Scenario $S_{13}$ : mean and randomly chosen sample path for the population, the effort and the profit per unit time. The optimal variable effort policy is on the left side and the optimal constant effort sustainable policy is on the right side. 

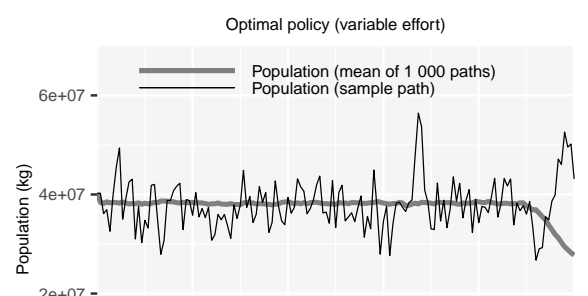

$0 \mathrm{e}+00-$
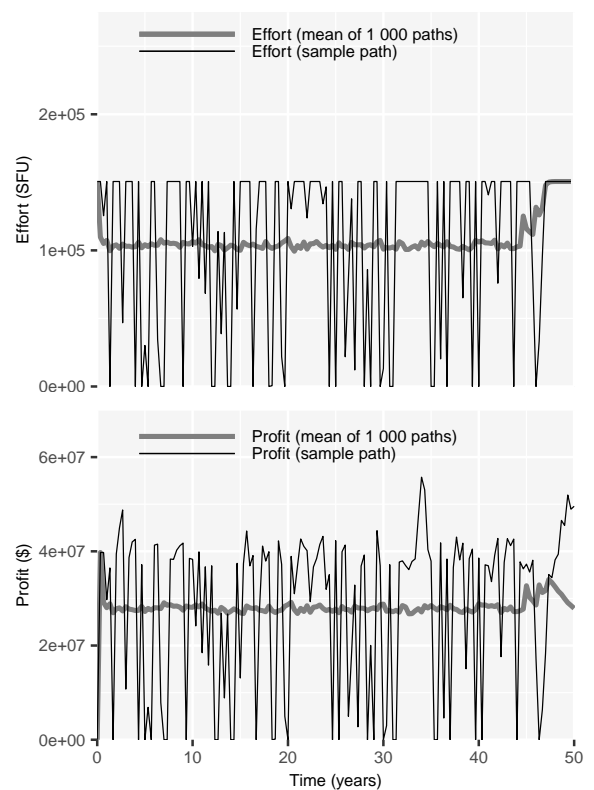

Optimal sustainable policy (constant effort)

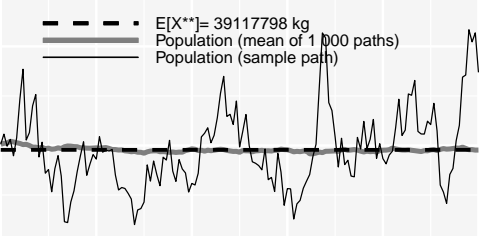

$\longrightarrow E^{* *}=104541 \mathrm{SFU}$

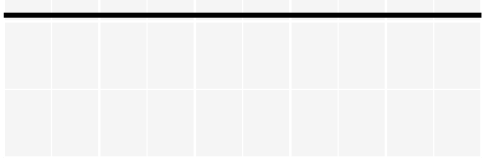

- - - $E\left[P^{* *}\right]=\$ 26820384$ Profit (mean of 1000 paths)
Profit (sample path)

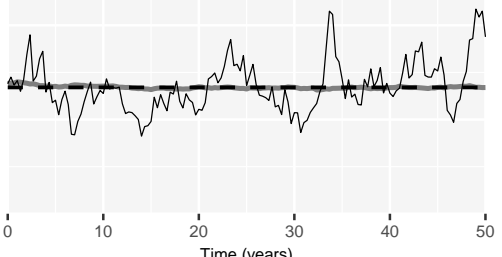

Figure 14: Scenario $S_{14}$ : mean and randomly chosen sample path for the population, the effort and the profit per unit time. The optimal variable effort policy is on the left side and the optimal constant effort sustainable policy is on the right side.

Note: Figures for the scenarios $S_{15}, S_{16}, S_{17}$ and $S_{18}$ are not shown since they are almost undistinguishable from the figure associated to the basic scenario $S_{0}$. 


\section{$2 \mathrm{R}$ code for computations and graphics}

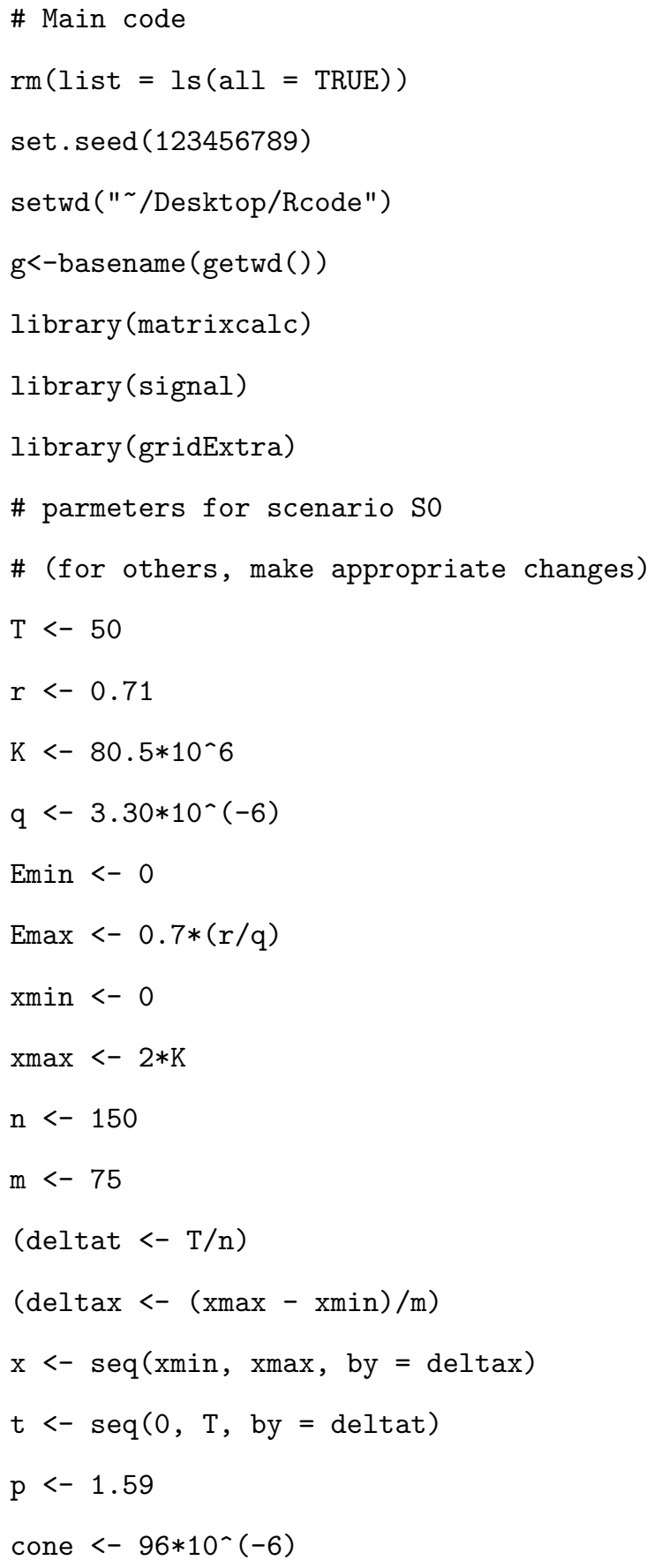




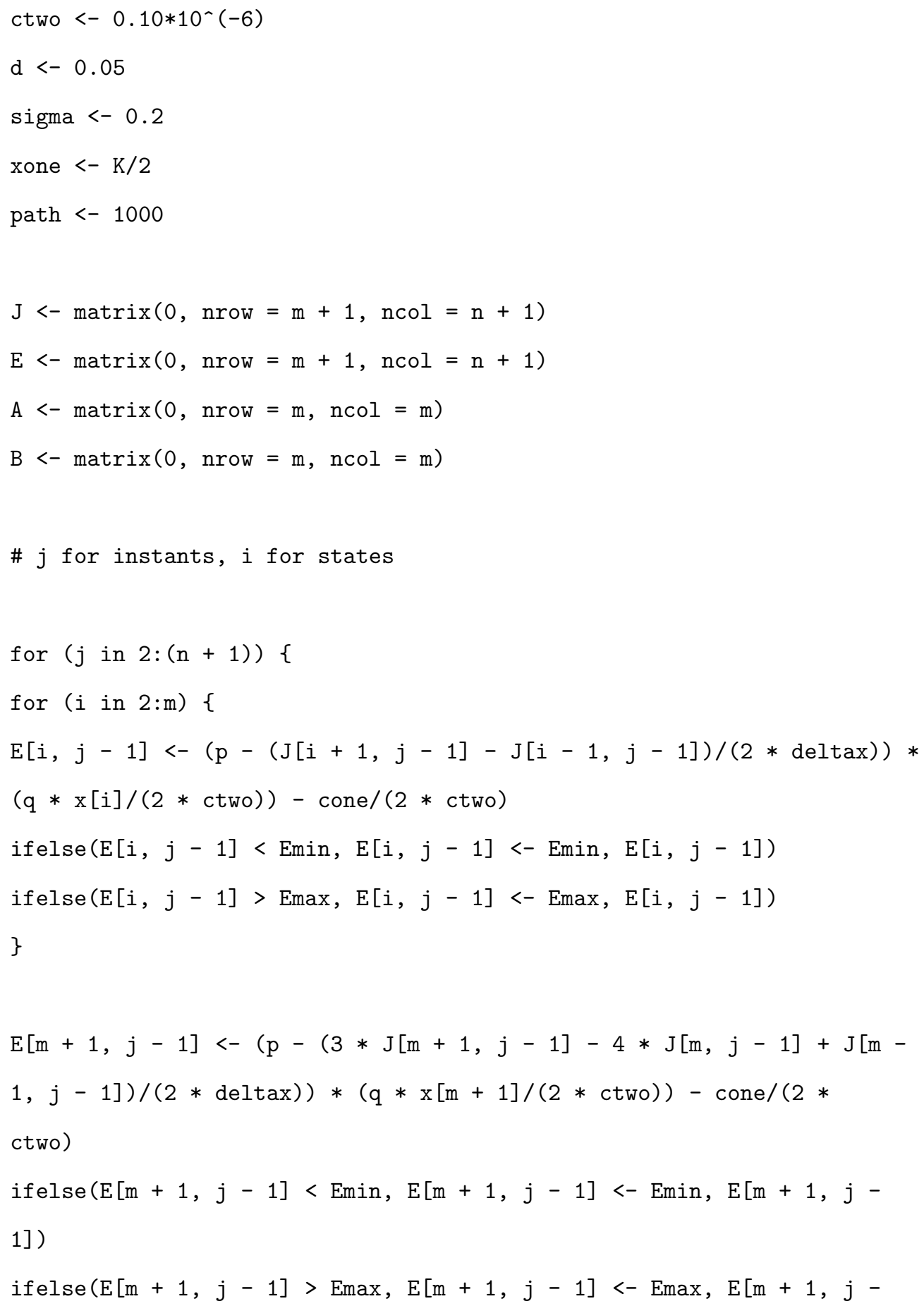


1])

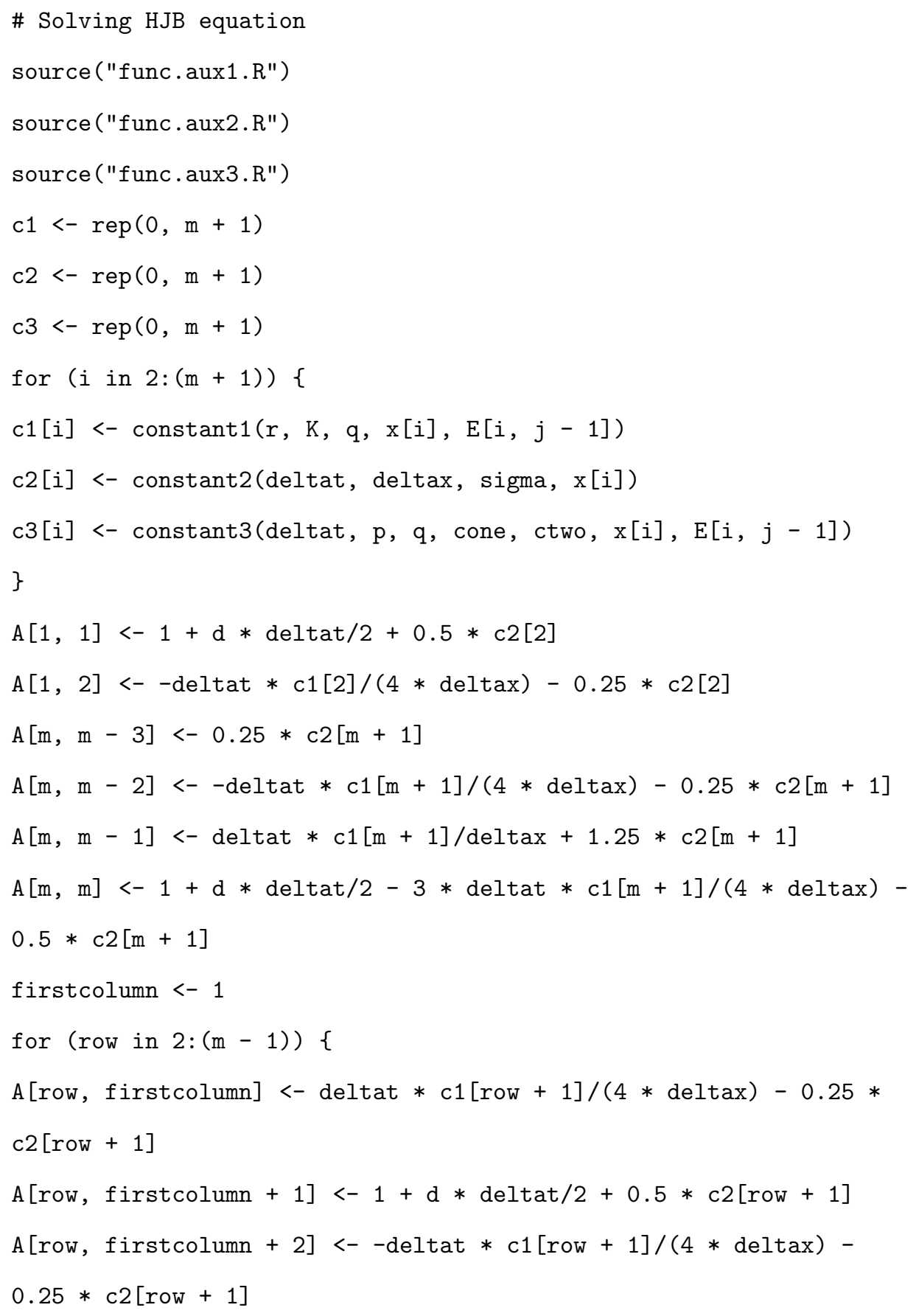




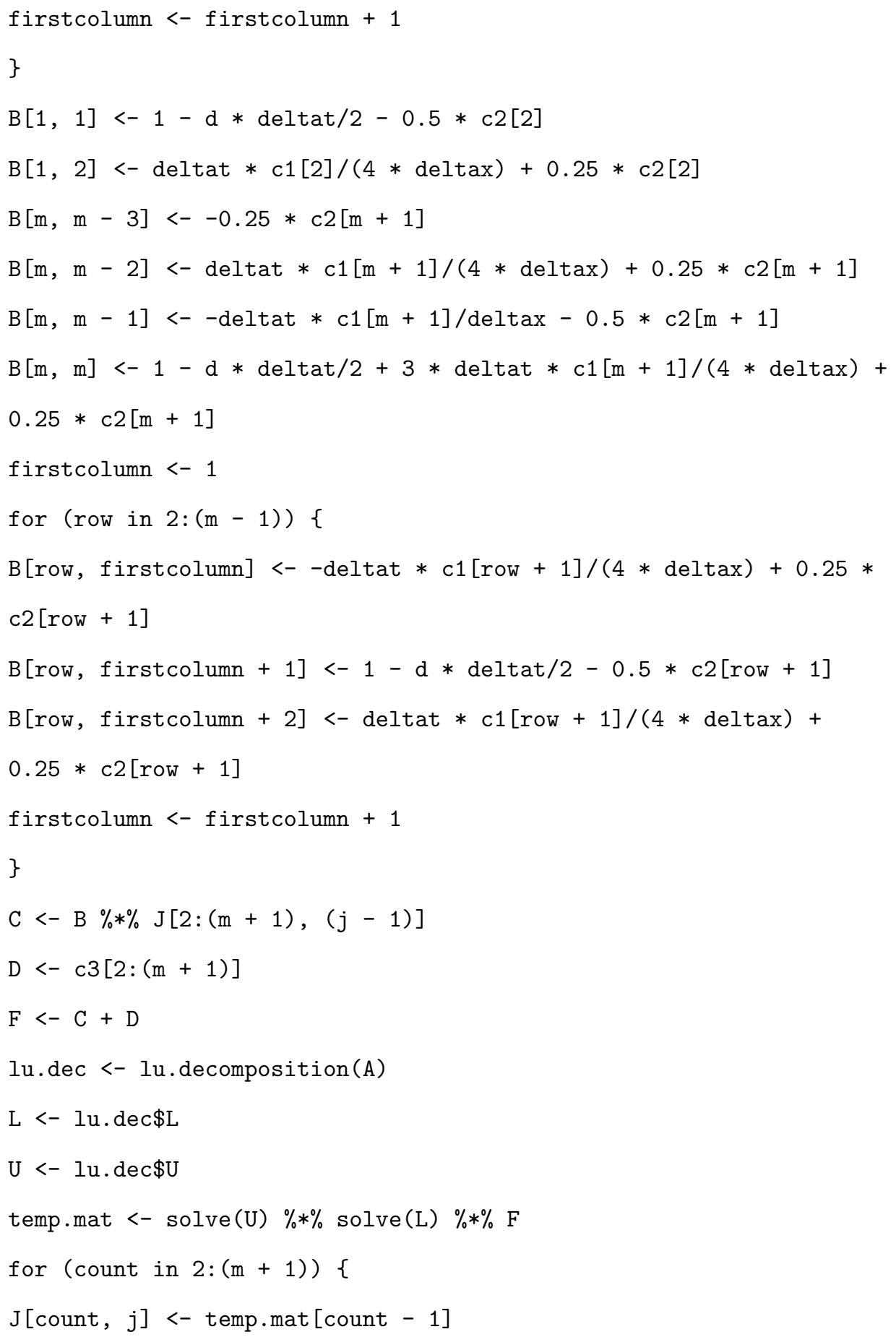




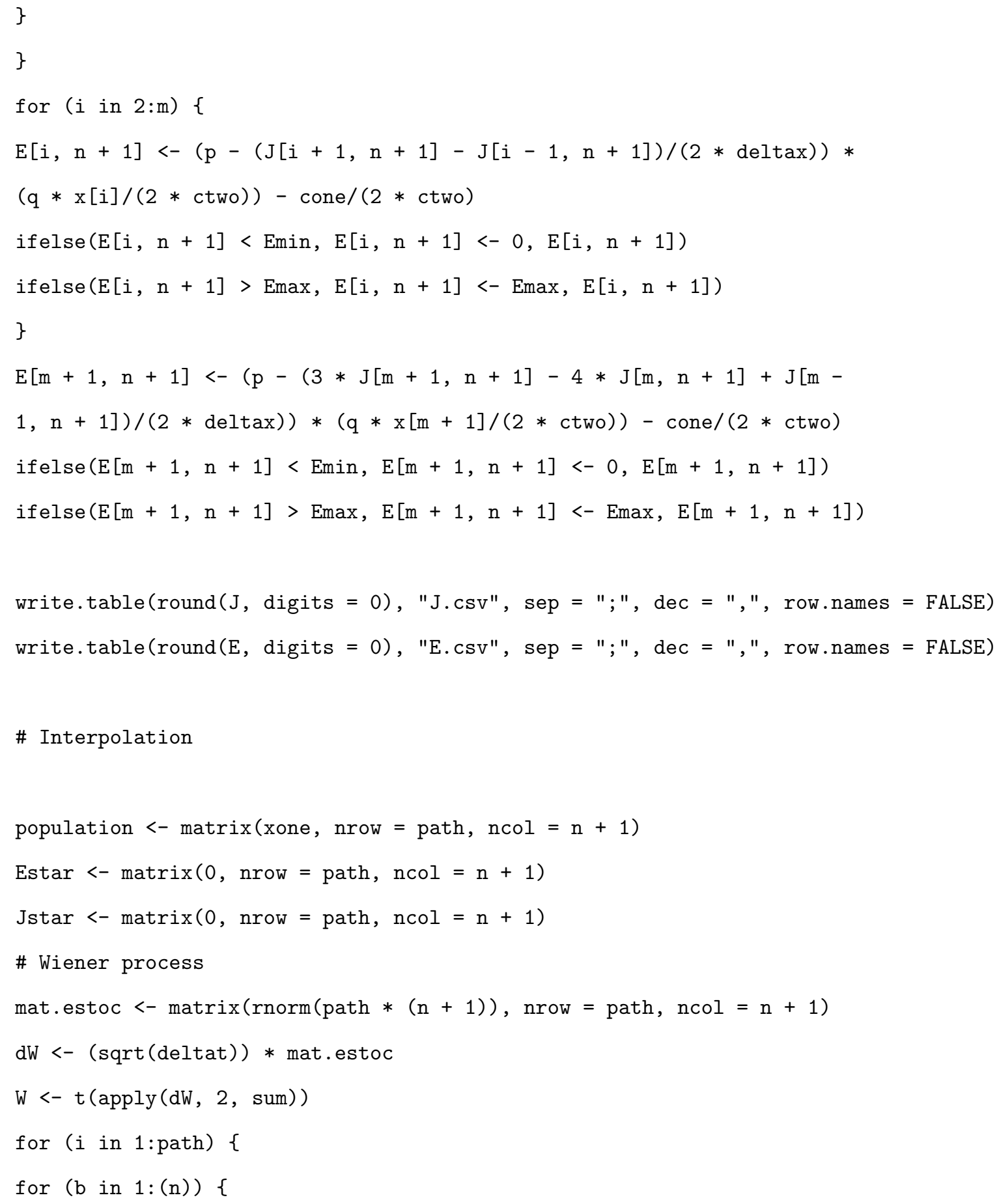




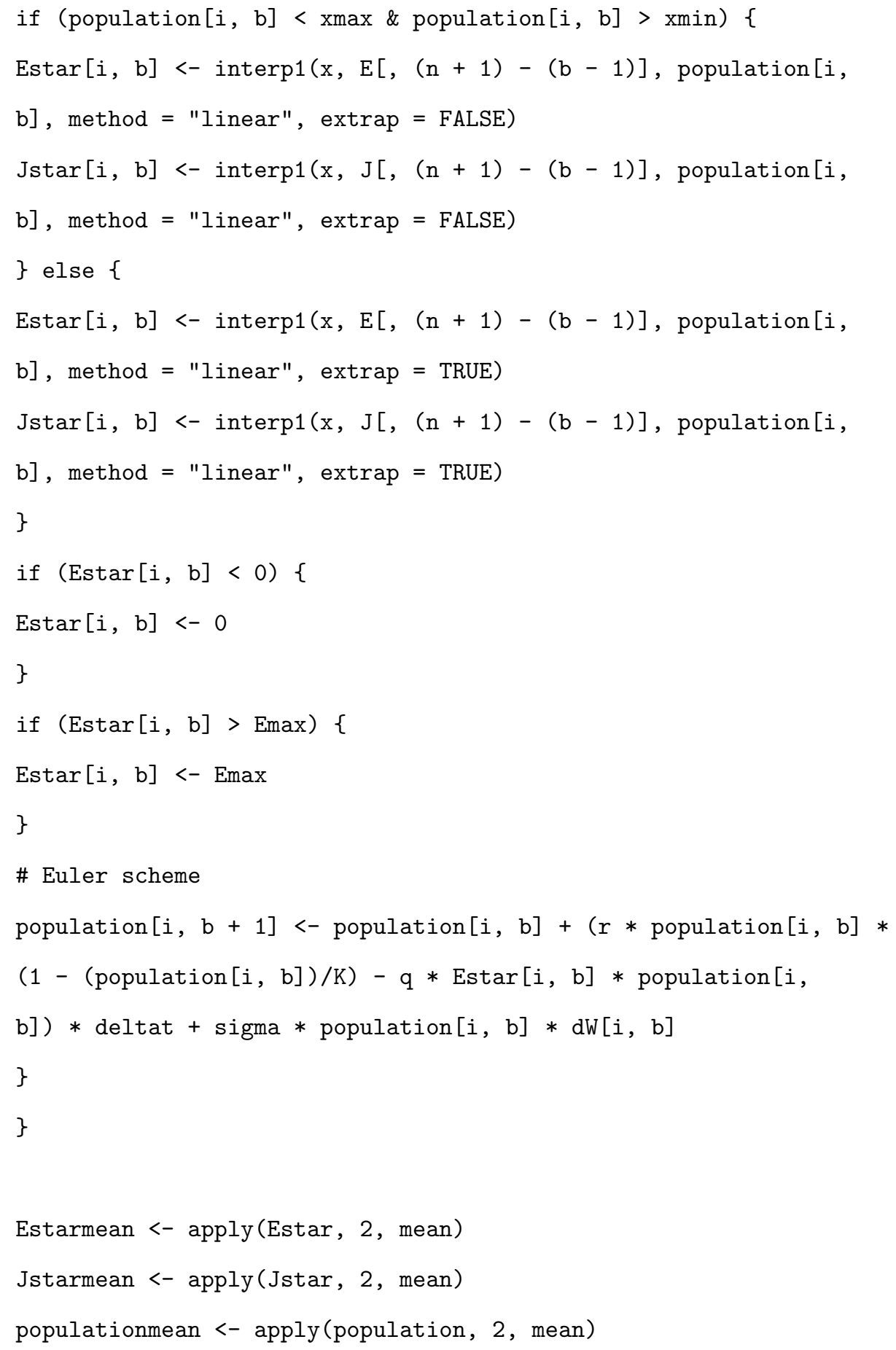




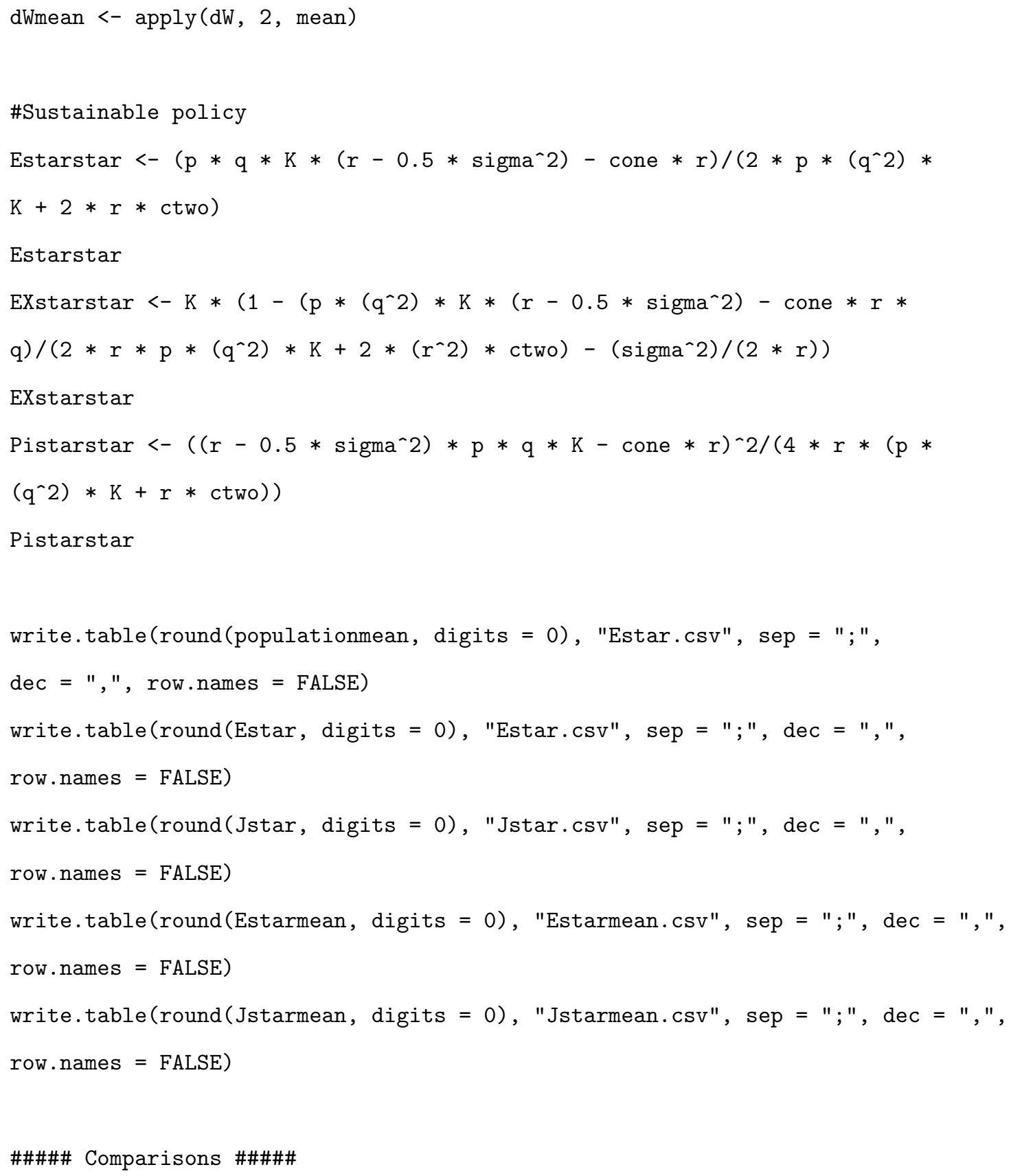




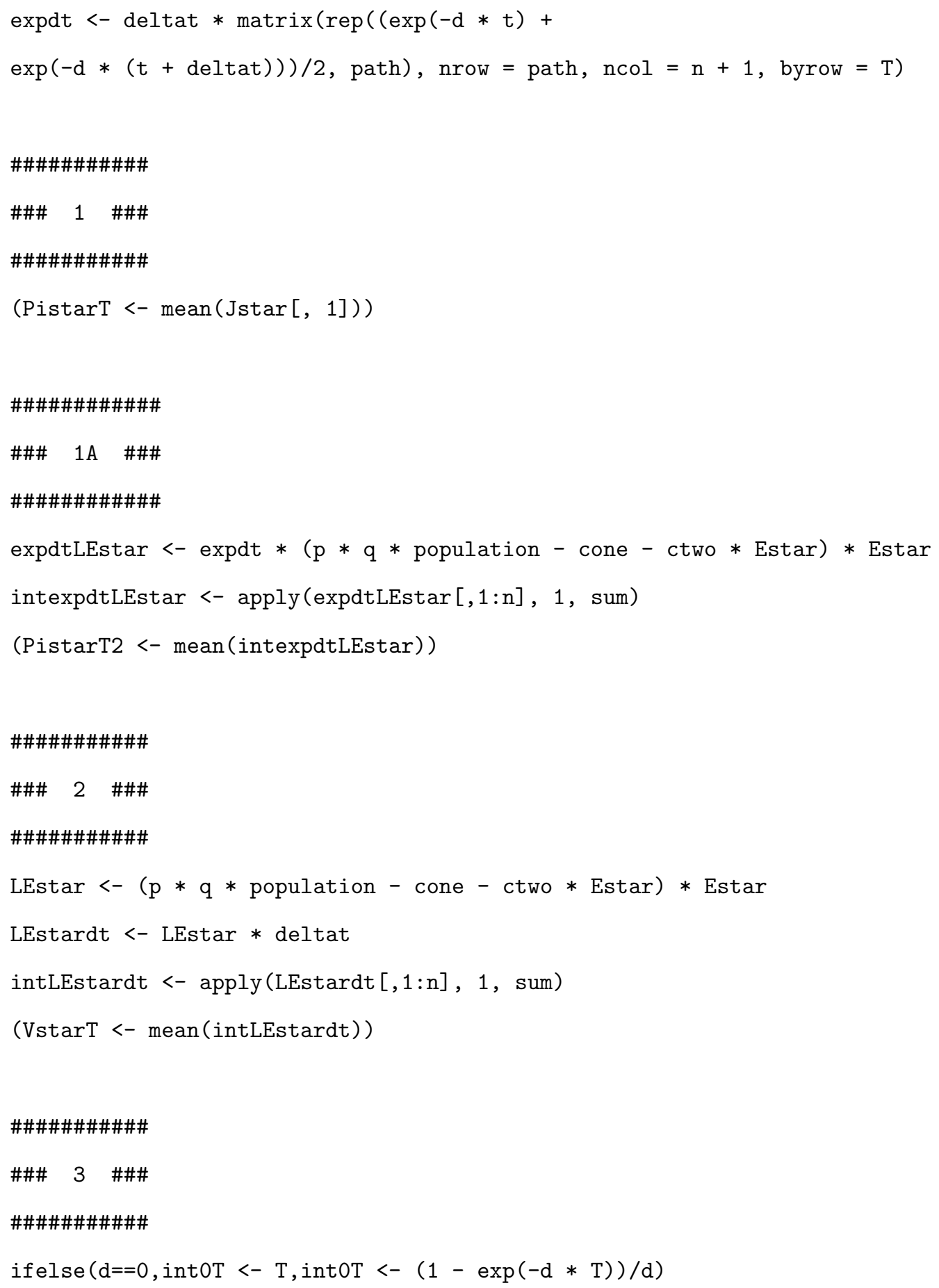


(PistarT2/int0T)

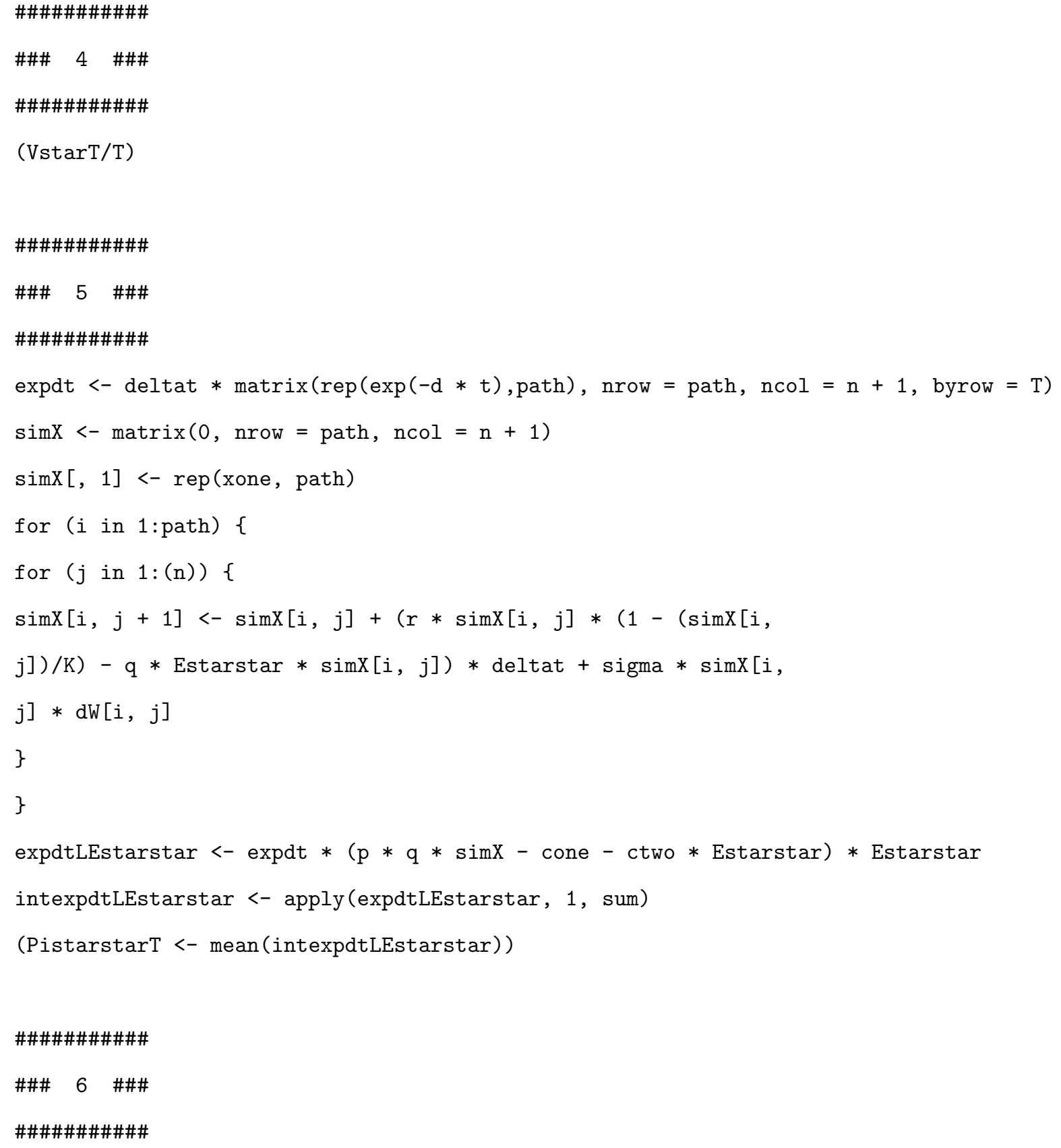




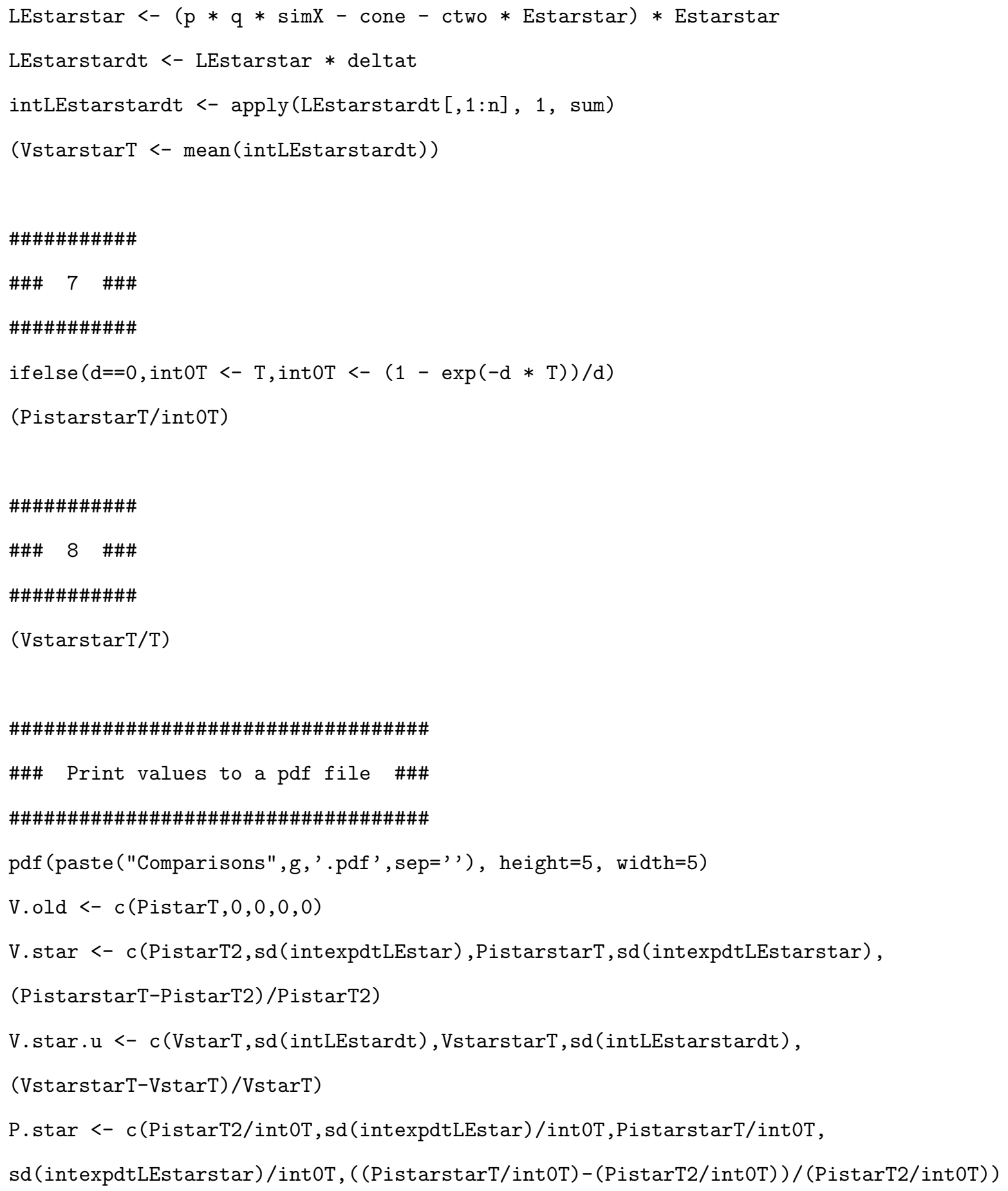




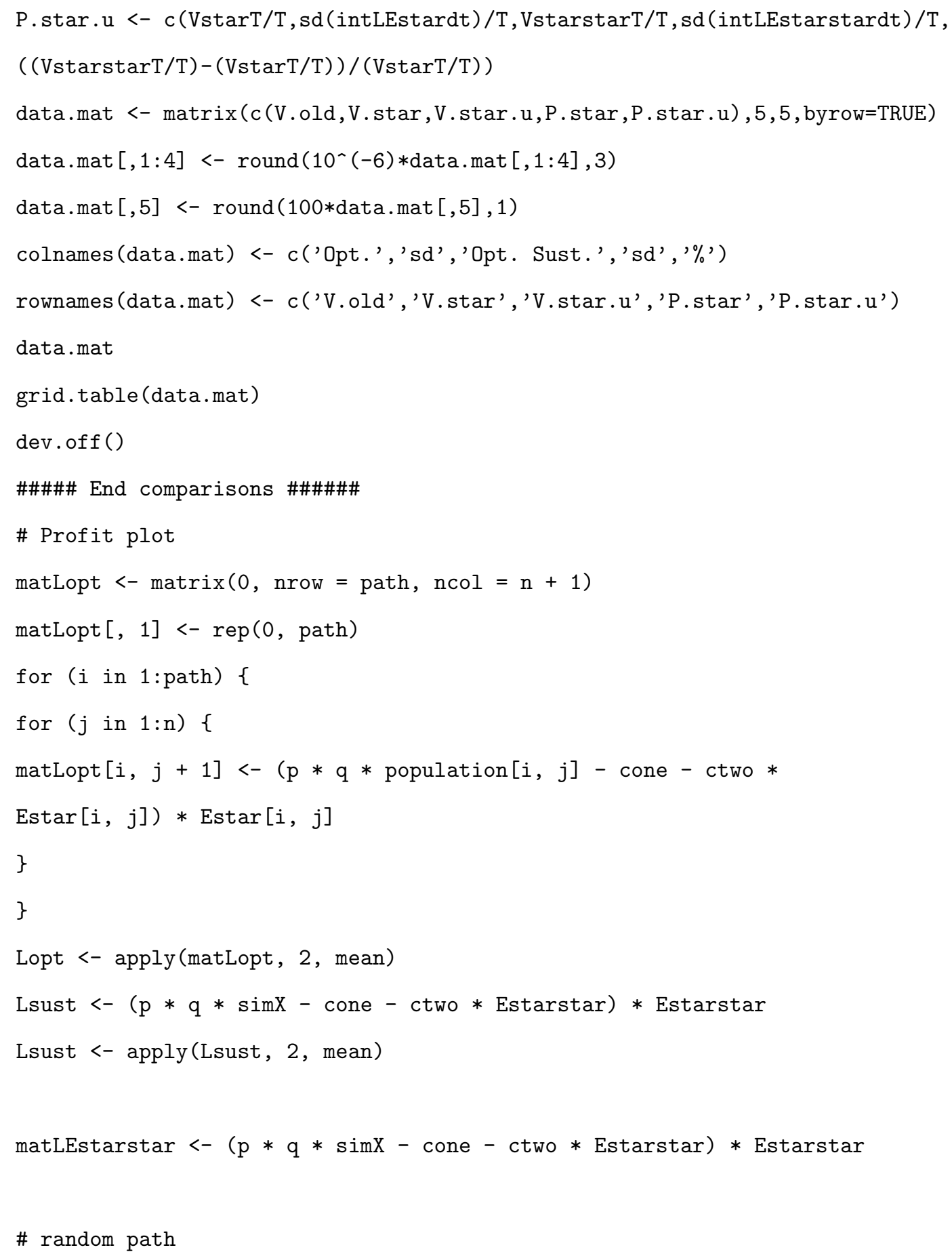


$($ num $1<-$ floor (runif $(1) *$ path $)+1)$

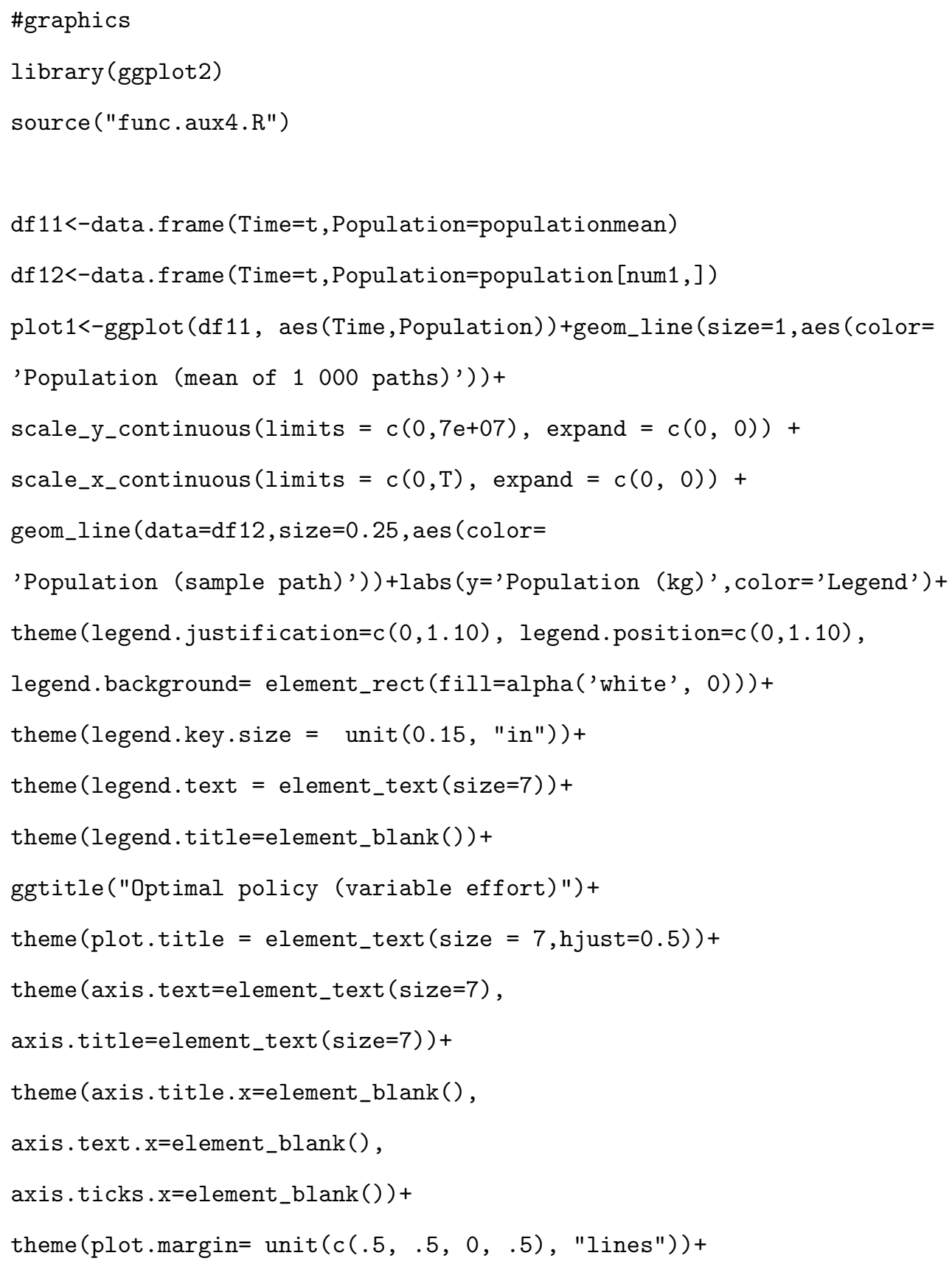




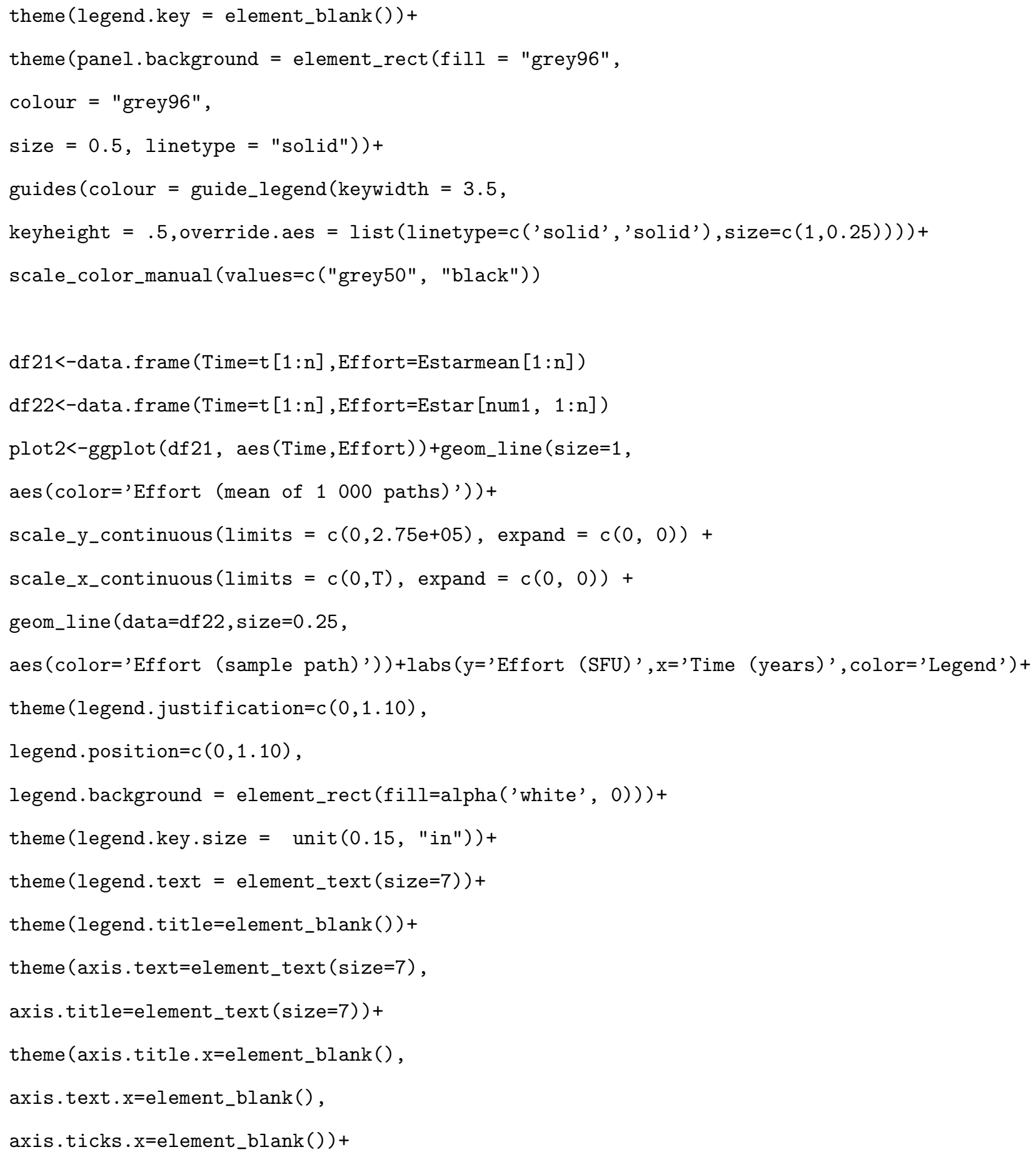




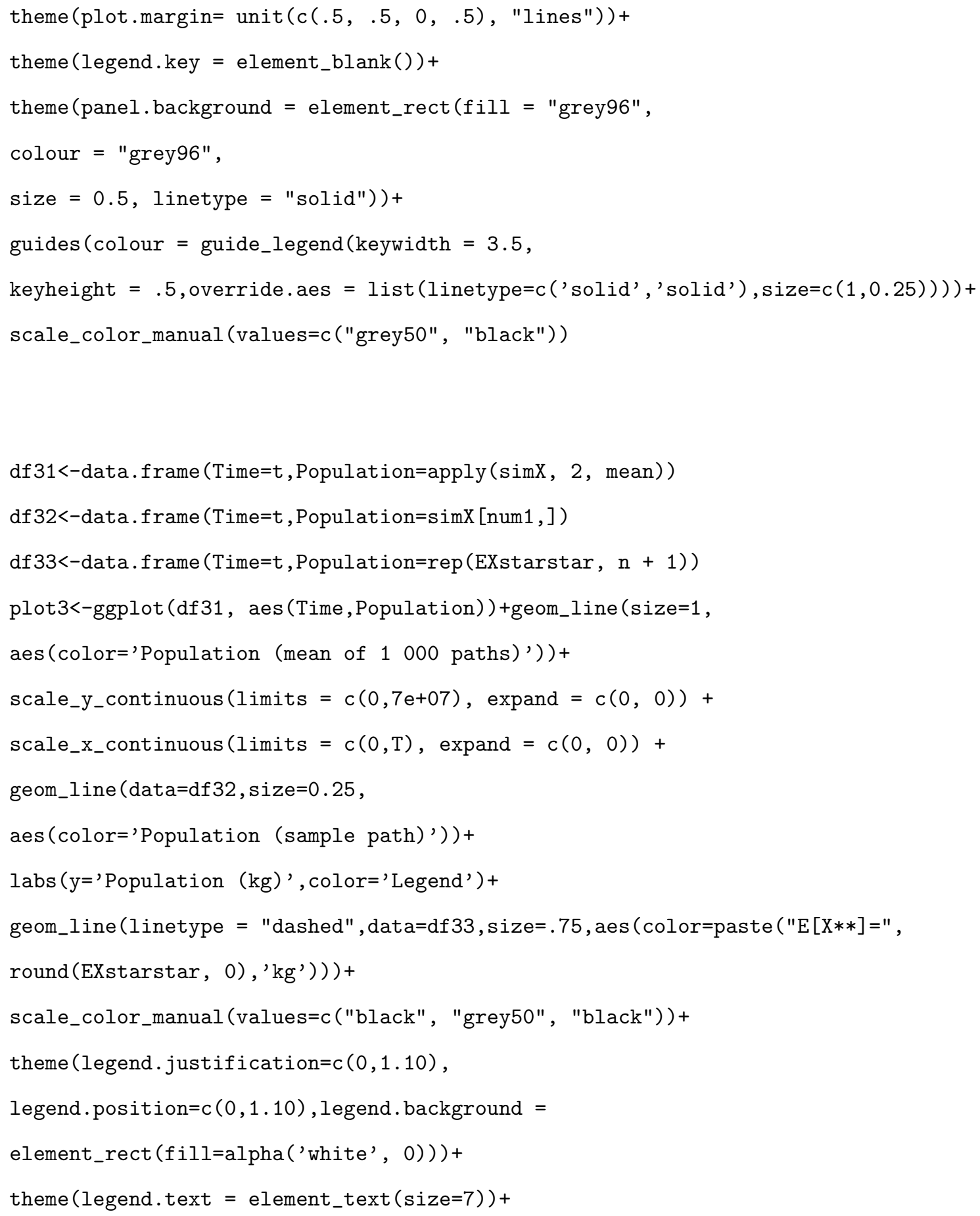




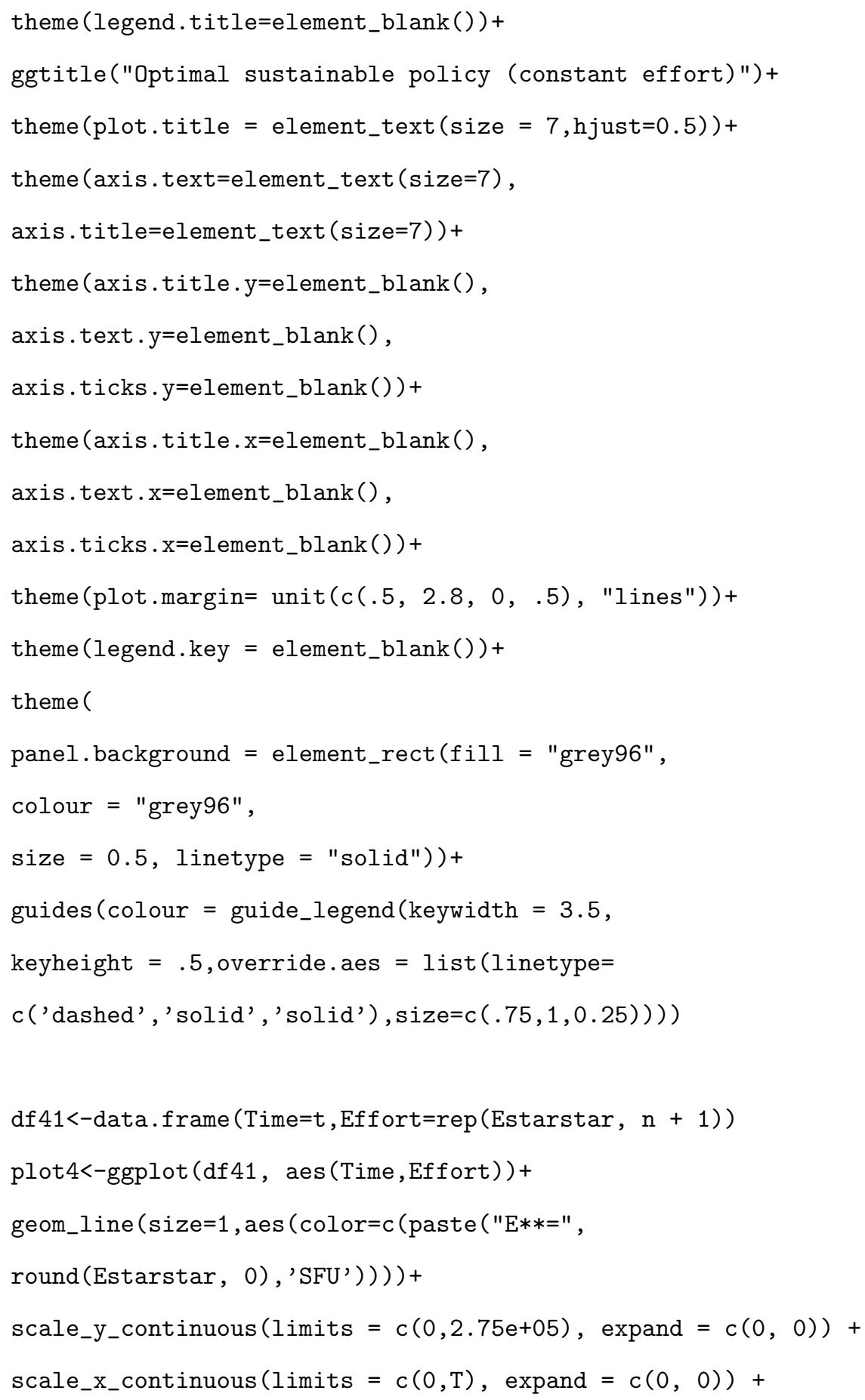




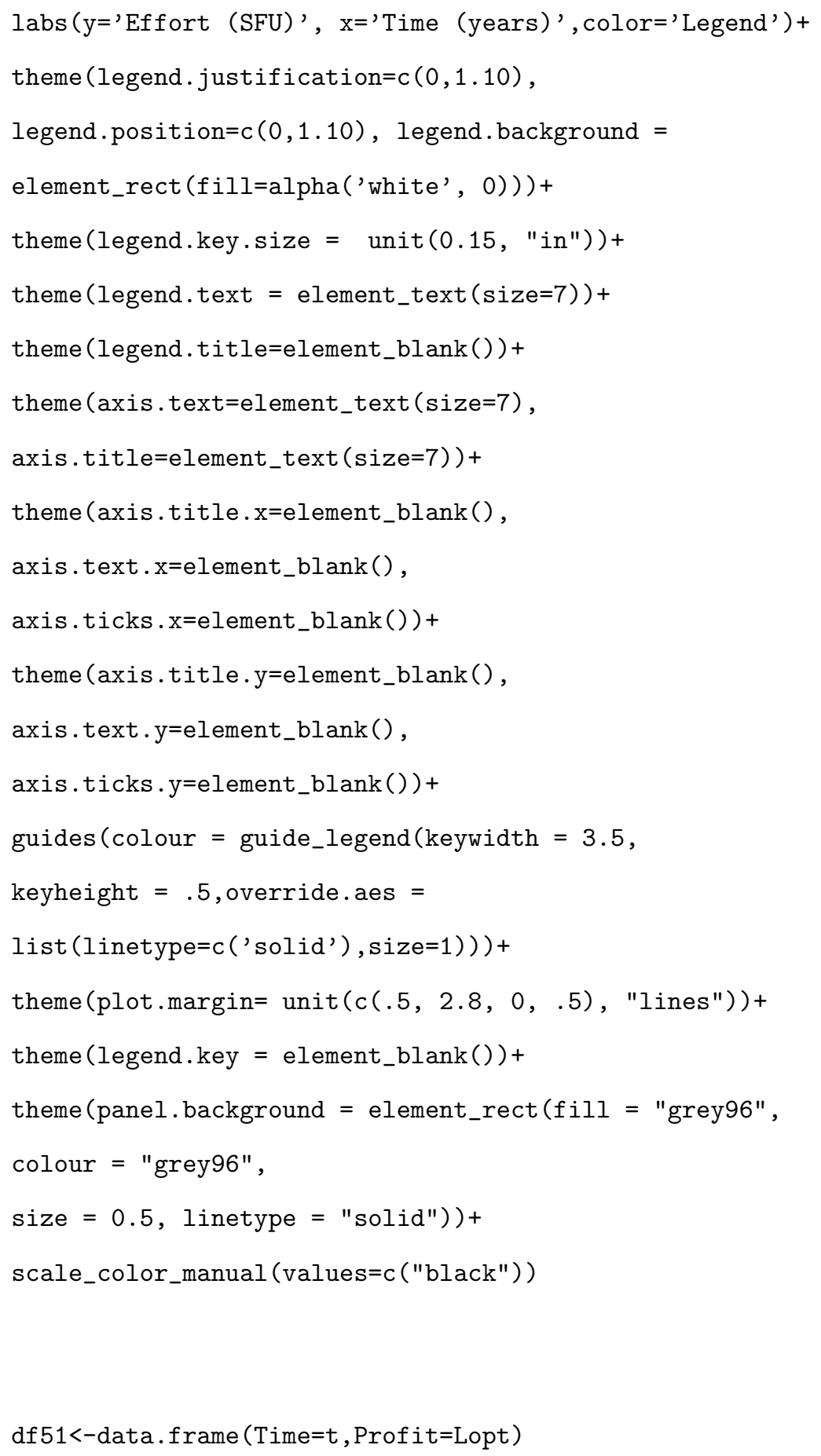




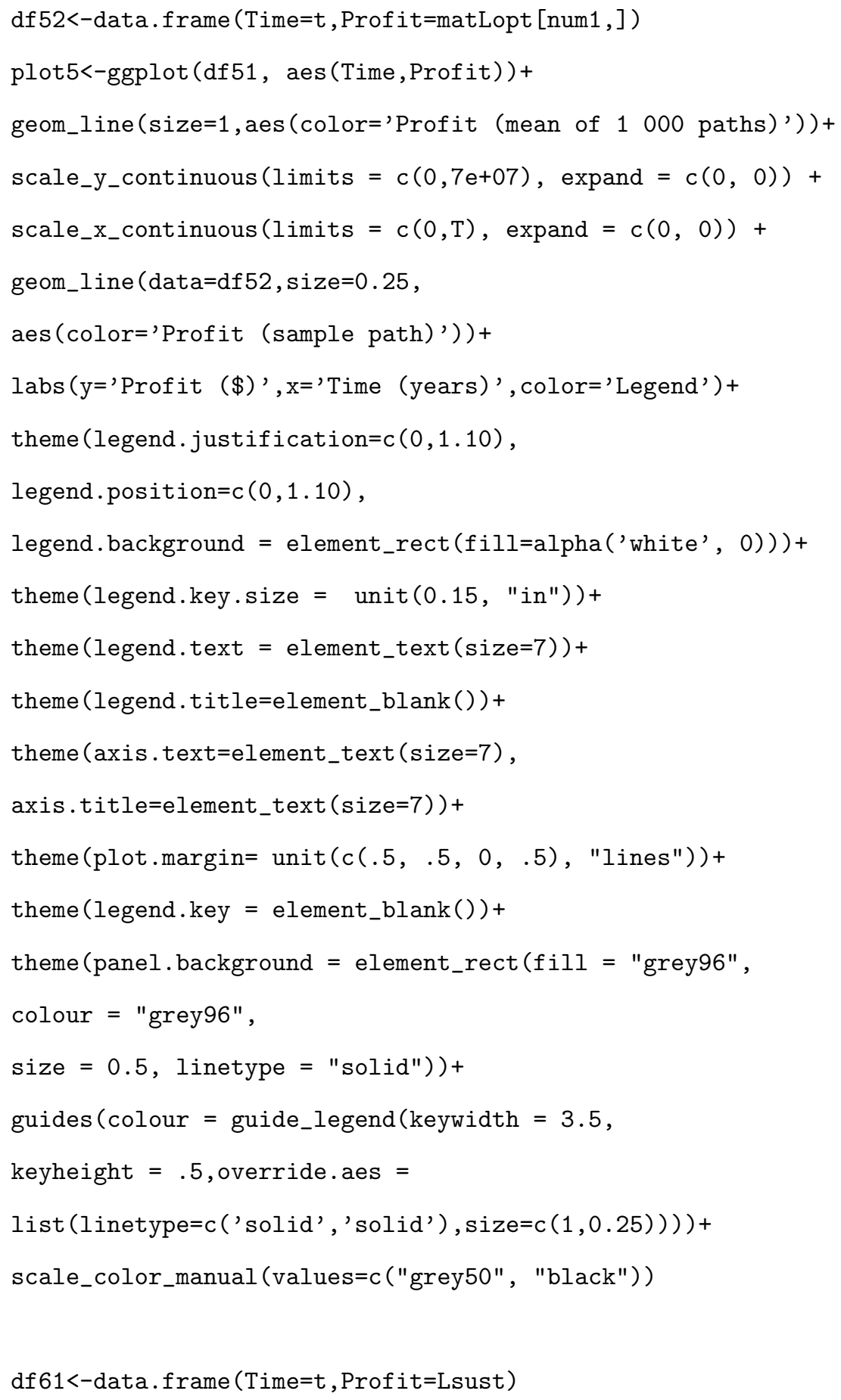




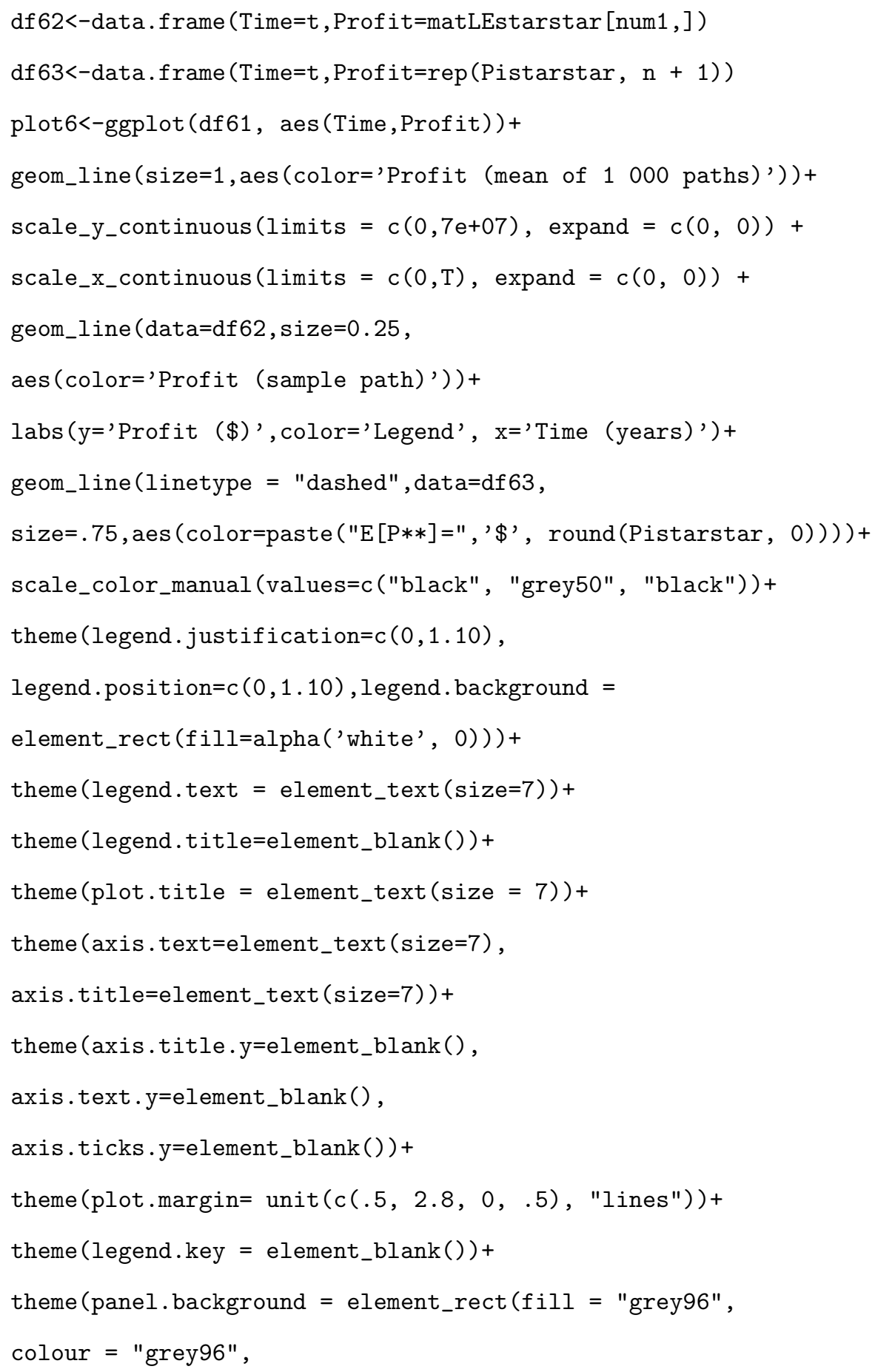




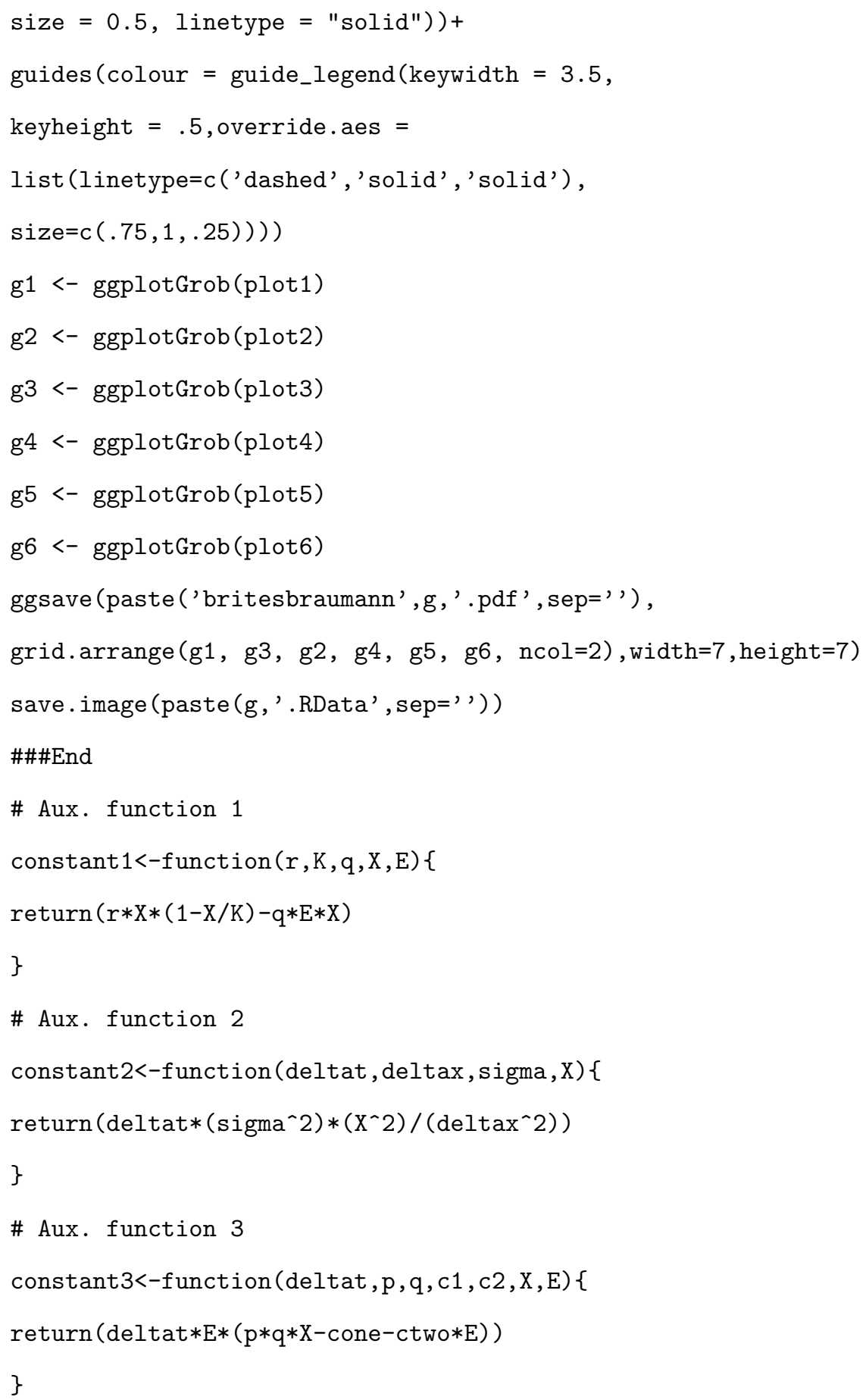




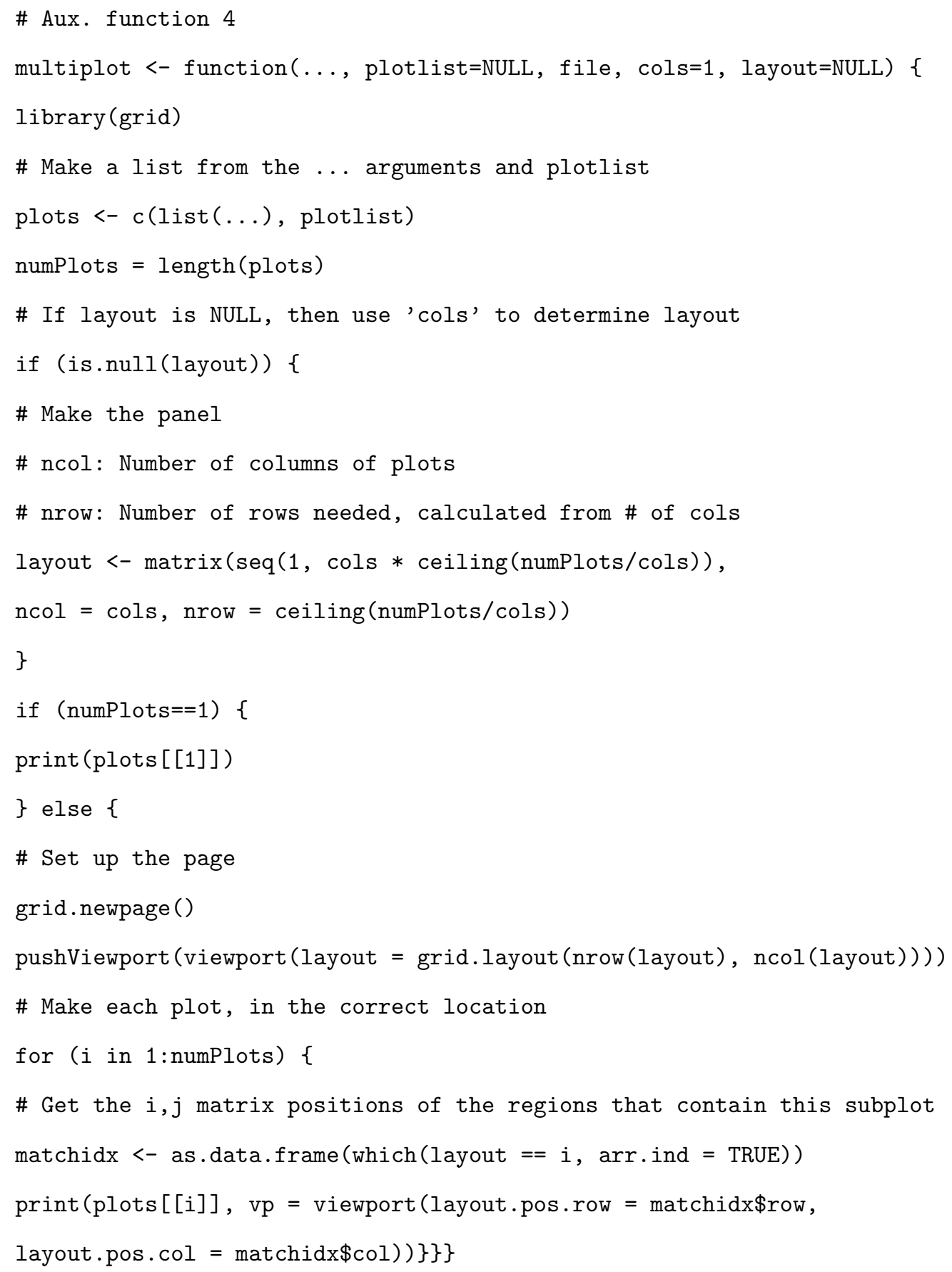

